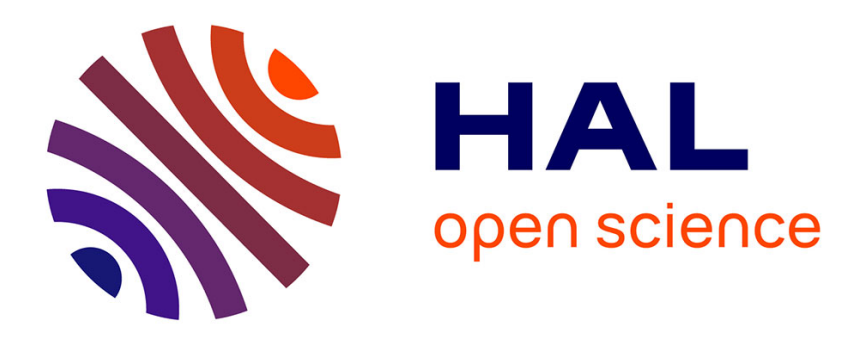

\title{
Inelasticity corrections for time-of-flight and fixed wavelength neutron diffraction experiments
}

Alan K Soper

\section{To cite this version:}

Alan K Soper. Inelasticity corrections for time-of-flight and fixed wavelength neutron diffraction experiments. Molecular Physics, 2009, 107 (16), pp.1667-1684. 10.1080/00268970903025667 . hal00513296

\section{HAL Id: hal-00513296 \\ https://hal.science/hal-00513296}

Submitted on 1 Sep 2010

HAL is a multi-disciplinary open access archive for the deposit and dissemination of scientific research documents, whether they are published or not. The documents may come from teaching and research institutions in France or abroad, or from public or private research centers.
L'archive ouverte pluridisciplinaire HAL, est destinée au dépôt et à la diffusion de documents scientifiques de niveau recherche, publiés ou non, émanant des établissements d'enseignement et de recherche français ou étrangers, des laboratoires publics ou privés. 


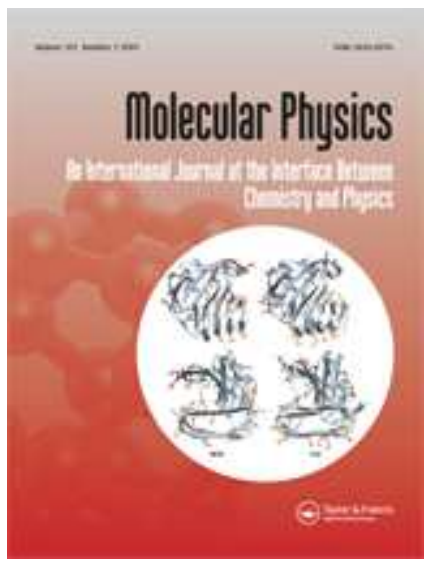

\section{Inelasticity corrections for time-of-flight and fixed wavelength neutron diffraction experiments}

\begin{tabular}{|c|c|}
\hline Journal: & Molecular Physics \\
\hline Manuscript ID: & TMPH-2009-0084.R1 \\
\hline Manuscript Type: & Full Paper \\
\hline $\begin{array}{r}\text { Date Submitted by the } \\
\text { Author: }\end{array}$ & 24-Apr-2009 \\
\hline Complete List of Authors: & Soper, Alan; STFC Rutherford Appleton Laboratory, ISIS Facility \\
\hline Keywords: & $\begin{array}{l}\text { inelasticity corrections, neutron diffraction, molecular liquids, } \\
\text { molecular glasses }\end{array}$ \\
\hline \multicolumn{2}{|c|}{$\begin{array}{l}\text { Note: The following files were submitted by the author for peer review, but cannot be converted } \\
\text { to PDF. You must view these files (e.g. movies) online. }\end{array}$} \\
\hline TOFinelastic.tex & \\
\hline
\end{tabular}

\section{s ScholarONE" \\ Manuscript Central}




\title{
Inelasticity corrections for time-of-flight and fixed wavelength neutron diffraction experiments
}

\author{
A.K.Soper \\ STFC Rutherford Appleton Laboratory, ISIS Facility, \\ Harwell Science and Innovation Campus, Didcot, Oxon, OX11 0QX, UK
}

(Dated: April 24, 2009) 


\begin{abstract}
Time-of-flight neutron diffraction methods are widely used to study the structure of liquids and glasses. The scattering nuclei in these experiments suffer nuclear recoil in the course of the neutron scattering process, which gives rise to distortions to both the self and distinct structure factors extracted from the data. These distortions are in general difficult to evaluate quantitatively, especially when the mass of the nucleus is similar to that of the neutron, such as when hydrogen is present in the material being studied. Traditional treatments of this effect generally assume the neutron energy is lower than the excitation energy of an atom, but for time-of-flight diffraction this is never the case, and the experiments typically sample a wide range of energy transfers from sub$\mathrm{meV}$ to tens of $\mathrm{eV}$. In addition, by attempting to produce an analytical correction, such methods invariably make a long list of approximations which can be difficult to justify in particular cases. Here a model for the scattering kernal is developed based on the well known harmonic oscillator model (A C Zemach and R J Glauber, Phys. Rev. 101, 118 (1956)). This is shown to have the correct first and second moments of the scattering kernal for both the self and distinct scattering, and is used to estimate the self and distinct scattering from a diatomic "dumbell" molecule. The model gives a realistic account of the single atom scattering from light and heavy water over a wide range of incident neutron energies, but is not yet accurate enough to perform quantitative corrections. In lieu of a quantitative approach, a "top hat" convolution method is developed to perform the subtraction of self scattering from real data, and to allow data from multiple detector banks to be merged into a single structure factor. The harmonic oscillator model is also used to address the question of inelasticity effects on the interference scattering. For the intramolecular correlations at least at low scattering angles up to $\sim 40^{\circ}$ it is found that the effect of inelasticity is rather small - around $0.6 \%$ on the estimated $\mathrm{OH}$ bond length for $\mathrm{H}_{2} \mathrm{O}$. Although the emphasis here is on time-of-flight diffraction, the model is quite general and can just as easily be applied to the case of fixed wavelength neutron diffraction where it also gives accurate results.
\end{abstract}




\section{INTRODUCTION}

Inelasticity effects occur widely in neutron diffraction experiments and arise because the energy exchanged when a neutron is scattered by a material can be as large as the energy of the neutron itself. They are particularly significant when scattering from light atoms such as hydrogen and deuterium, when, due to the mass equivalence, large amounts of energy can be exchanged in the scattering process. Loss (or gain) in energy by the scattering radiation means that, since energy and momentum must be conserved in the scattering process, the scattered particle also changes momentum, so that the ideal of integrating the dynamic scattering law, $S(Q, \epsilon)$ at constant $Q$, where $\hbar Q$ is the momentum change and $\epsilon$ is the energy change in the scattering process, to yield the so-called "static" structure factor, $S(Q)$, [1] can never be obtained in practice. This effect has been known about for a long time and was first studied in detail by Placzek [2], who showed that in the event that the average amount of energy exchanged with the scattering system was small compared to the typical excitation energies of that system, and the mass of the neutron was much smaller than that of the scattering atom, then a simple correction could be derived based on the ratio of the mass of the neutron to the mass of the scattering atom, and the first and second moments of $S(q, \epsilon)$. Unfortunately neither condition is valid for light atoms such as hydrogen, deuterium or helium, for which systems the Placzek correction is completely inappropriate.

The subject has been studied extensively by numerous other authors such as [3-29] but to date there has been no completely satisfactory way of performing this correction for light atoms, in spite of Powles optimistic title [11] "Slow-neutron scattering by molecules .5. Recoil corrections for any molecule". The various approaches fall into three main categories: those which derive from the original moment expansion as proposed by Placzek [2], for example $[5-11,13-15,20,21,23]$, those that use a model for $S(Q, \epsilon)$ and integrate this over the relevant path in momentum and energy transfer space, for example [12, 16-19, 22, 25-28], and those that use some form of ad hoc fitting function to remove the single atom scattering, for example [24, 29, 30].

It is not the present intention to assess all these different approaches in detail, but it is clear that none of the methods can be claimed to work quantitatively for materials containing light atoms, and that the method of fitting a functional form to the single atom scattering may inadvertently remove genuine structural information from the data as well. Apart from 
the fact that the Placzek method introduces unacceptably large approximations for light atoms, there is the additional problem that both incident neutron spectrum and detector efficiency vary widely in a time-of-flight (TOF) neutron experiment, and there is no way these variations can be modelled correctly in any approximate scheme. Hence, as new neutron instrumentation is developed with the promise of higher neutron fluxes and wider ranges of momentum transfer, it is imperative that a rigorous and reliable method be found for removing, or at least ameliorating as much as possible, the effects of inelasticity in neutron diffraction data.

The present paper is devoted to this task, and has as its core the development a realistic dynamical scattering law, $S(Q, \epsilon)$, based on the exact solution for a harmonic oscillator, which is applicable over a very wide range of $(Q, \epsilon)$, which can be used to simulate both single atom and interference scattering, and which is integrated over the correct trajectory for any given experimental configuration. To date the results are very encouraging, but probably not yet accurate enough to perform a quantitative correction on data from a range of detectors at different scattering angles, so that currently it is still necessary to invoke an ad hoc functional form to remove residual effects in TOF diffraction data. A new method of doing this using a so-called "top hat" function is described which may help to remove some of the bias that can be present in other methods. For reactor experiments the current approach appears already accurate enough to remove inelasticity effects from materials containing light hydrogen without resorting to this second stage.

Water diffraction data are used to demonstrate the method for both the time-of-flight and reactor data, but this was primarily one of convenience because the relevant data were readily to hand and previously published. Ultimately the method is intended for any molecular system that can be reduced to a suitable number of vibrational excitations.

\section{THEORY OF TIME-OF-FLIGHT DIFFRACTION}

The example used here for time-of-flight diffraction will be the SANDALS diffractometer based at ISIS, which is specifically designed for looking at materials containing hydrogen, and has neutron detectors up to scattering angles of $\sim 40^{\circ}$. If $k_{i}$ is the incident neutron wavevector and $k_{f}$ is the scattered neutron wavevector, then conservation of momentum and energy in the nuclear collision require that the momentum transfer, $\hbar Q$, and energy transfer, 
$\epsilon$, are given by

$$
\begin{aligned}
Q^{2} & =k_{i}^{2}+k_{f}^{2}-2 k_{i} k_{f} \cos \theta \\
\epsilon & =\frac{\hbar^{2}}{2 m}\left(k_{i}^{2}-k_{f}^{2}\right)
\end{aligned}
$$

where $m$ is the mass of the neutron. For a fixed wavelength diffraction experiment $k_{i}$ is defined by the incident beam monochromator, but for a time-of-flight (TOF) experiment the incident and final wavevectors are determined from the requirement that all neutrons that arrive at the detector in a given time channel have the same time-of-flight from the source. This constraint is satisfied by the condition

$$
\frac{1+R}{k_{e}}=\frac{1}{k_{i}}+\frac{R}{k_{f}}
$$

where $\mathrm{R}$ is the ratio of scattered to incident flight paths and $k_{e}$ is the wavevector at zero energy transfer, i.e. $k_{i}=k_{f}$. Therefore $k_{e}$ is determined from the total time-of-flight for a particular channel, and we define the elastic momentum transfer as

$$
Q_{e}=2 k_{e} \sin \left(\frac{\theta}{2}\right)
$$

Diffraction data from glasses and liquids are normally reported as a function of this elastic $\mathrm{Q}$ value, but it should be remembered they actually contain contributions from a range of $\mathrm{Q}$ values and energy transfers. It also should be noted that the fixed wavelength condition is recovered when $R=0$, i.e. zero scattered flight path, since then it takes no time for the neutrons to travel to the detector, so the time-of-flight fixes the incident wavevector, $k_{i}=k_{e}$.

Fig. 1 shows the trajectory in $(Q, \epsilon)$ space sampled by a time-of-flight neutron diffraction experiment on SANDALS at a scattering angle of $30.71^{\circ}$ over a range of $Q_{e}$ values. Also shown is the recoil energy of a proton. The latter represents approximately the centroid of the scattering kernal for the single atom scattering of a proton, irrespective of whether it is bound to a molecule or not. (This was originally shown by Placzek in 1952 [2]). It can be seen that a very wide range of $Q$ and $\epsilon$ is sampled under all time-of-flight conditions. In particular the experiment explores energy transfers which go into $10 \mathrm{~s}$ of eV which is well above any likely molecular vibration energies and enters the region of electronic excitations and molecular dissociation. At high $Q_{e}$ the trajectories approach the ideal constant $\mathrm{Q}$ 
trajectory in the region of the recoil energy, but at low $Q_{e}$ the trajectories become highly curved near $Q=Q_{e}$. Hence one might expect that inelasticity corrections are larger at low $Q_{e}$ in the TOF experiment, as indeed is observed.

The double differential scattering cross section for a single atom for scattering into unit solid angle and per unit energy transfer was shown by van Hove (1954) to be equal to $b^{2} \frac{k_{f}}{k_{i}} S(Q, \epsilon)$, where $S(Q, \epsilon)$ is the dynamic scattering law and $b$ is the scattering length of the atom. The diffraction scattering cross section obtained in a TOF experiment is therefore an integral of this double differential cross section along paths of constant TOF, such as are shown in Fig. 1. Because both the incident and final wavevectors vary along this path, and because the incident flux of neutrons depends on the incident wavevector, while the detection efficiency depends on the final wavevector, the integral to be evaluated is non-trivial (see for example Powles 1993 or Egelstaff 1987):

$$
\frac{d \sigma}{d \Omega}=\overline{b^{2}} \int_{\text {const.TOF }} \frac{\Phi\left(k_{i}\right)}{\Phi\left(k_{e}\right)}\left(\frac{\partial k_{i}}{\partial k_{e}}\right)_{\epsilon} \frac{D\left(k_{f}\right)}{D\left(k_{e}\right)} \frac{k_{f}}{k_{i}} S(Q, \epsilon) d \epsilon
$$

It is assumed here that the raw data have been divided by the incident neutron flux, $\Phi\left(k_{i}\right)$, and detector efficiency, $D\left(k_{f}\right)$, at the elastic wavevector, $k_{e}$. The bar over the square of the neutron scattering length, $b$, represents the average over the spin and isotope states of the atom. The Jacobian $\left(\frac{\partial k_{i}}{\partial k_{e}}\right)_{\epsilon}$ is required to allow for the uneven sampling of the incident spectrum for different values of $\epsilon$ along a path of constant TOF. It is straightforward to show, using equations 2 and 3 , that

$$
\left(\frac{\partial k_{i}}{\partial k_{e}}\right)_{\epsilon}=\frac{k_{i}^{2}}{k_{e}^{2}} \frac{1+R}{1+R\left(\frac{k_{i}}{k_{f}}\right)^{3}}
$$

Although the integral (5) can be awkward to integrate, depending on the nature of the dynamic scattering law, there is no in principle difficulty with doing this since all the other terms in the kernal are either well known or can be estimated sufficiently accurately to give confidence in the final diffraction cross section. The only quantity that is not well defined is the dynamic scattering law itself, $S(Q, \epsilon)$, since this is genarally an unknown at the outset.

A number of approximate methods exist, based on a Taylor expansion about $Q=Q_{e}$ (see for example Placzek 1952, Powles 1973), to estimate the effect of inelasticity on the diffraction pattern without detailed knowledge of the dynamic scattering law. These are generally useful when the mass of the scattering atom is much larger than the mass of the 


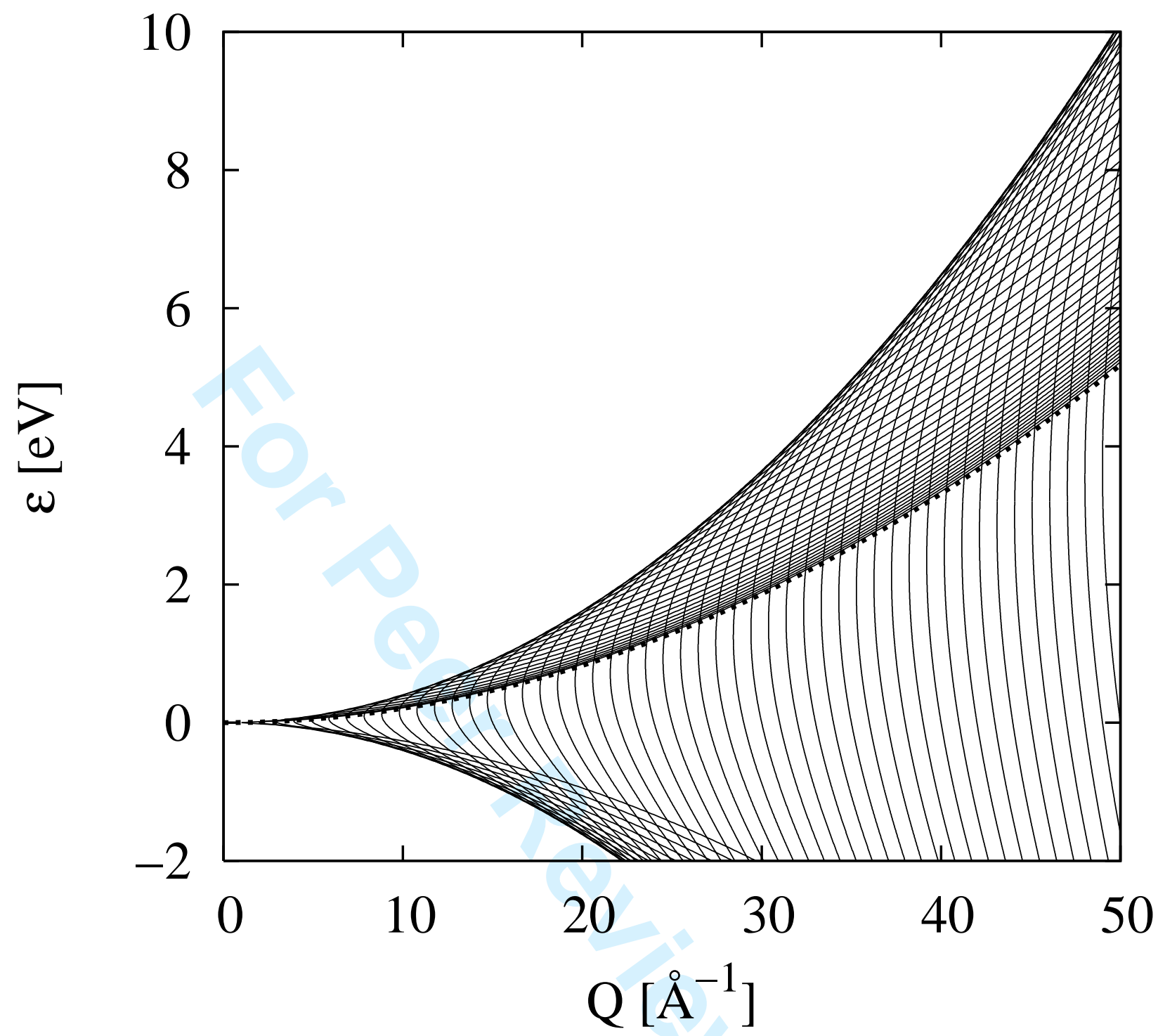

FIG. 1: Constant time-of-flight trajectories sampled in a time-of-flight neutron diffraction experiment on SANDALS at a scattering angle of $30.71^{\circ}$. The flight path ratio $R$ is 0.134 in this case. Trajectories are shown for 50 values of $Q_{e}$ in the range $0-50 \AA^{-1}$ in steps of $1 \AA^{-1}$. The dashed line shows the recoil energy of a proton: this is the centroid of the self scattering kernal for a hydrogen atom. The upper and lower limits on the energy transfer for any given $\mathrm{Q}$ value are set by the kinematic equations, 1 and 2, and the scattering angle of the detector: as the scattering angle is made smaller, these lines become further apart, and the $(Q, \epsilon)$ trajectories become more parallel to the $\epsilon$-axis, implying that the ideal experiment would measure the diffraction pattern at low scattering angles and high incident neutron energy, conditions that are difficult to realise in practice. Negative energy transfers are mostly not sampled due to the detailed balance factor in the dynamic scattering law. 
neutron, but they do not work for light atoms like hydrogen and deuterium. Hence for light atoms there is no choice but to perform the integral (5) numerically using a model scattering law, combined with estimates of the incident neutron spectrum and detector efficiency function.

\section{THE HARMONIC OSCILLATOR SCATTERING LAW}

In order to make an estimate of the effect of inelasticity on the diffraction pattern for both the it is therefore necessary make an assumption for $S(Q, \epsilon)$. This scattering law must satisfy several constraints. It must satisfy the known moment conditions for both self and distinct scattering - see Placzek [2]. It must satisfy detailed balance, namely $S(Q,-\epsilon)=$ $\exp \left(-\frac{\epsilon}{k T}\right) S(Q, \epsilon)$. It must work for a system where there may be several excitation modes (of angular frequency $\omega_{\lambda}$ ) with $\frac{\hbar \omega_{\lambda}}{k T}<<1$ to $\frac{\hbar \omega_{\lambda}}{k T}>>1$. Finally it should ideally be analytic over the full range of energy and momentum transfers, such as shown in Fig. 1, in order to make numerical solution of the integral possible. Although there have been several attempts at defining such a scattering law they all fail in one or more of these requirements.

The only one that survives detailed scrutiny is that of the harmonic oscillator, orginally due to Zemach and Glauber [31]. Even this cannot accurately represent the scattering from a complex molecular system over the full energy range, but it does at least allow us to investigate the effect of a range of excitations at different frequencies on the diffracted scattering cross section, and has the correct limiting form at high $Q$ and $\epsilon$. For a general, harmonically oscillating assembly of atoms, they showed that the double differential scattering cross-section for scattering into unit solid angle at wavevector change $\mathbf{Q}$ and energy transfer $\epsilon$ is given by a product of oscillator functions, one for each normal coordinate of the oscillating system:

$$
\frac{d^{2} \sigma}{d \Omega d \epsilon}=\frac{k_{f}}{k_{i}} \sum_{\nu, \nu^{\prime}} \overline{b_{\nu} b_{\nu^{\prime}}}\left\langle\exp \left[i \mathbf{Q} \cdot\left(\mathbf{r}_{\nu}-\mathbf{r}_{\nu^{\prime}}\right)\right] \prod_{\lambda} W_{\nu \nu^{\prime}}^{(\lambda)}\right\rangle_{\Omega}
$$

where

$$
W_{\nu \nu^{\prime}}^{(\lambda)}=\exp \left\{-\hbar\left[\left(\mathbf{Q} \cdot \mathbf{c}_{\nu}^{(\lambda)}\right)^{2}+\left(\mathbf{Q} \cdot \mathbf{c}_{\nu^{\prime}}^{(\lambda)}\right)^{2}\right] \frac{\operatorname{coth} y_{\lambda}}{4 \omega_{\lambda}}\right\} \exp \left(n_{\lambda} y_{\lambda}\right) I_{n_{\lambda}}\left(\frac{\hbar\left(\mathbf{Q} \cdot \mathbf{c}_{\nu}^{(\lambda)}\right)\left(\mathbf{Q} \cdot \mathbf{c}_{\nu^{\prime}}^{(\lambda)}\right)}{2 \omega_{\lambda} \sinh y_{\lambda}}\right)
$$


and the orientational average in (7) is performed over the orientations of $\mathbf{Q}$ with respect to the molecular axes. Here, $b_{\nu}, \mathbf{r}_{\nu}$, and $\mathbf{c}_{\nu}^{(\lambda)}$ are the scattering length, position vector, and normal coordinate of mode $\lambda$ with angular frequency $\omega_{\lambda}$, for atom $\nu$, respectively. The bar over the product $b_{\nu} b_{\nu^{\prime}}$ represents the average over the spin and isotope states of each atom, and note that the result of this average will in general be different for the single atom scattering $\left(\nu=\nu^{\prime}\right)$ compared to the interference scattering $\left(\nu \neq \nu^{\prime}\right) . y_{\lambda}=\hbar \omega_{\lambda} / 2 k T$. The energy transfer $\epsilon=\sum_{\lambda} n_{\lambda} \hbar \omega_{\lambda}$, with $n_{\lambda}$ the change in excitation quantum number for mode $\lambda$. (Note that the sign of the energy transfer (2) used here is the opposite to that used by Zemach and Glauber [31].)

For a general assembly of $N$ atoms there will be $D N$ degrees of freedom, where $D$ is the dimensionality of the system. In what follows we will assume that all motions of an array of atoms can be represented as vibrational in form, via harmonic oscillator functions. This is not an unreasonable assumption for atoms in the condensed state since they will inevitably be affected by forces from surrounding atoms and so will never be truly free particles. This approximation will break down at low $Q$ and at long times (small $\epsilon$ when diffusion and other non-oscillatory motions may be present, but otherwise it should give a realistic representation of the dynamic scattering law. It also will not correctly represent any anharmonicity in the force field, but as will be seen it has the correct limiting form when the temperature is much higher than the vibrational frequency, which will be generally true for translational modes, and it has the correct limiting form at high $Q$ when from the impulse approximation [32] the experiment samples the atomic momentum distribution of the atom prior to excitation. A particular and important result is that this scattering law has the correct first moment for both the self and interference scattering at all $Q$ values.

The simplest possible case that includes interference scattering is the diatomic molecule. We assume the masses of the two atoms are $m_{1}$ and $m_{2}$. For this system in one dimension, there will be one translational mode and one vibrational mode. In two dimensions, there will be two translational modes along the $x$ and $y$ axes, and one librational mode perpendicular to the $x$ axis and one vibrational mode along the $x$ axis. In three dimensions there will be three translational modes, one along each of the $x, y$, and $z$ axes, two librational (about the $x$ and $y$ axes perpendicular to the interatomic $z$ axis) and one vibrational mode along the $z$ axis. The amplitude vectors are defined so that the displacement of each atom is written as [31] $\mathbf{u}(t)=\sum_{\lambda} \mathbf{c}_{\lambda} q(t)$, with $q_{\lambda}(t)$ the normal coordinate of mode $\lambda$, which represents the 
displacement of a 1-dimensional oscillator of unit mass. Hence, because the potential energy of mode $\lambda$ is expressed as $\frac{1}{2} \omega_{\lambda}^{2} q_{\lambda}^{2}(t)$, the amplitude vectors have the units of $1 / \sqrt{\text { mass }}$ with the normalisation [4] that for a given mode

$$
\sum_{\nu} M_{\nu}\left[c_{\nu}^{(\lambda)}\right]^{2}=1
$$

Since translational motion involves both atoms moving as a unit, the mass associated with this motion is $M=m_{1}+m_{2}$, so the amplitude vector is simply

$$
\mathbf{c}_{\nu}^{(\lambda)}=\frac{1}{\sqrt{M}}
$$

for all $\nu$ and for all axes. For the rotational and vibrational modes these occur relative to the centre of mass, leading to the requirement that

$$
\mathbf{c}_{1}^{(\lambda)}=-\frac{m_{2}}{m_{1}} \mathbf{c}_{2}^{(\lambda)}
$$

Combining (11) with (9) the solution is

$$
\begin{aligned}
& \mathbf{c}_{1}^{(\lambda)}=\sqrt{\frac{m_{2}}{m_{1} M}} \\
& \mathbf{c}_{2}^{(\lambda)}=-\sqrt{\frac{m_{1}}{m_{2} M}}
\end{aligned}
$$

for all rotational and vibrational modes and along all three axes.

A further simplification can be made at this point. The interest here is on 3-dimensional systems, which even with the present most simple model of a diatomic model would leave 6 modes to be summed over. However if some of those modes have the same frequency and the corresponding normal coordinates have the same magnitude as in the present example, then, because the harmonic oscillator scattering law consists of a product of exponential functions, the exponents of modes with the same frequency can be added prior to thermal averaging (see equation 3.12 of Zemach and Glauber [31]). For the present purposes it will be assumed here that the three translational modes have the same frequency, $\omega_{T}$, and the two librational modes have the same frequency $\omega_{L}$. This will lead to a sum over just three modes, 1 translational, 1 librational and 1 vibrational. This is sufficient for the purposes of demonstrating what this does to the differential cross-section, but may not be sufficient 
for quantitative agreement with measured data. However it is always be possible to remove this simplification to allow for more modes. For the translational modes the exponential and Bessel function arguments in (8)

$$
\sum_{\lambda=T}\left(\mathbf{Q} \cdot \mathbf{c}_{\nu}^{(\lambda)}\right)\left(\mathbf{Q} \cdot \mathbf{c}_{\nu^{\prime}}^{(\lambda)}\right)=Q^{2} c_{\nu}^{(T)} c_{\nu^{\prime}}^{(T)}
$$

since the sum of squares of direction cosines between $\mathbf{Q}$ and the molecule's axes add up to unity. The sum here is over the three translational modes. For the librational modes $\mu$ is the cosine of the angle between $\mathbf{Q}$ and the molecule's $z$ axis, which gives

$$
\sum_{\lambda=L}\left(\mathbf{Q} \cdot \mathbf{c}_{\nu}^{(\lambda)}\right)\left(\mathbf{Q} \cdot \mathbf{c}_{\nu^{\prime}}^{(\lambda)}\right)=Q^{2} c_{\nu}^{(L)} c_{\nu^{\prime}}^{(L)}\left(1-\mu^{2}\right)
$$

where the sum is over the 2 librational modes. For the vibrational mode the expression is

$$
\sum_{\lambda=V}\left(\mathbf{Q} \cdot \mathbf{c}_{\nu}^{(\lambda)}\right)\left(\mathbf{Q} \cdot \mathbf{c}_{\nu^{\prime}}^{(\lambda)}\right)=Q^{2} c_{\nu}^{(V)} c_{\nu^{\prime}}^{(V)} \mu^{2}
$$

where of course $c_{\nu}^{(V)}=c_{\nu}^{(L)}$.

\section{A. Moments of the scattering law}

The $k^{\text {th }}$ moment of the scattering law is defined as

$$
S_{k}=\int_{\text {const. Q }} \epsilon^{k} S(Q, \epsilon) d \epsilon
$$

which becomes for the discrete levels of the harmonic oscillator functions

$$
S_{k}^{\left(\nu \nu^{\prime}\right)}=\left\langle\exp \left[i \mathbf{Q} \cdot\left(\mathbf{r}_{\nu}-\mathbf{r}_{\nu^{\prime}}\right)\right] \prod_{\lambda}\left(\hbar \sum_{\lambda} n_{\lambda} \omega_{\lambda}\right)^{k} W_{\nu \nu^{\prime}}^{(\lambda)}\right\rangle_{\Omega}
$$

Making use of the well known sum rule on the Bessel functions [33],

$$
\exp \left[\frac{1}{2} x\left(y+\frac{1}{y}\right)\right]=\sum_{k=-\infty}^{+\infty} y^{k} I_{k}(x)
$$

it is possible to sum this expression analytically to give 


$$
\begin{aligned}
S_{0}^{\left(\nu \nu^{\prime}\right)} & =\left\langle X_{\nu \nu^{\prime}}\right\rangle_{\Omega} \\
S_{1}^{\left(\nu \nu^{\prime}\right)} & =\left\langle\frac{\hbar^{2} Q^{2}}{2} \sum_{\lambda}\left(\hat{\mathbf{Q}} \cdot \mathbf{c}_{\nu}^{(\lambda)}\right)\left(\hat{\mathbf{Q}} \cdot \mathbf{c}_{\nu^{\prime}}^{(\lambda)}\right) X_{\nu \nu^{\prime}}\right\rangle_{\Omega} \\
S_{2}^{\left(\nu \nu^{\prime}\right)} & =\left\langle\left\{\left[\frac{\hbar^{2} Q^{2}}{2} \sum_{\lambda}\left(\hat{\mathbf{Q}} \cdot \mathbf{c}_{\nu}^{(\lambda)}\right)\left(\hat{\mathbf{Q}} \cdot \mathbf{c}_{\nu^{\prime}}^{(\lambda)}\right)\right]^{2}+\frac{\hbar^{2} Q^{2}}{2} \sum_{\lambda}\left(\hat{\mathbf{Q}} \cdot \mathbf{c}_{\nu}^{(\lambda)}\right)\left(\hat{\mathbf{Q}} \cdot \mathbf{c}_{\nu^{\prime}}^{(\lambda)}\right) \hbar \omega_{\lambda} \operatorname{coth} y_{\lambda}\right\} X_{\nu \nu^{\prime}}\right\rangle_{\Omega}
\end{aligned}
$$

where

$$
X_{\nu \nu^{\prime}}=\exp \left[i \mathbf{Q} \cdot \mathbf{d}_{\nu \nu^{\prime}}\right] \exp \left[-\frac{\hbar^{2} Q^{2}}{2} \sum_{\lambda} \frac{\left[\hat{\mathbf{Q}} \cdot\left(\mathbf{c}_{\nu}^{(\lambda)}-\mathbf{c}_{\nu^{\prime}}^{(\lambda)}\right)\right]^{2} \operatorname{coth} y_{\lambda}}{2 \omega_{\lambda}}\right]
$$

$\mathbf{d}_{\nu \nu^{\prime}}$ is the interatomic separation vector, and $\hat{\mathbf{Q}}$ the unit vector in the direction of $\mathbf{Q}$. We note that orthnormality of the normal coordinates [34] requires that

$$
\frac{\hbar^{2} Q^{2}}{2} \sum_{\lambda}\left(\hat{\mathbf{Q}} \cdot \mathbf{c}_{\nu}^{(\lambda)}\right)\left(\hat{\mathbf{Q}} \cdot \mathbf{c}_{\nu^{\prime}}^{(\lambda)}\right)=\frac{\hbar^{2} Q^{2}}{2 M_{\nu}} \delta_{\nu \nu^{\prime}}
$$

identically whatever the molecular orientation with respect to $\mathbf{Q}$.

For the self terms, $\nu=\nu^{\prime}$ the atomic separation is zero and the amplitude vectors in $(23)$ are identical for every mode, so $X_{\nu \nu^{\prime}}=1$ and the moments become:

$$
\begin{aligned}
S_{0}^{(\nu)} & =1 \\
S_{1}^{(\nu)} & =\frac{\hbar^{2} Q^{2}}{2 m_{\nu}} \\
S_{2}^{(\nu)}-\left[S_{1}^{(\nu)}\right]^{2} & =\left\langle\frac{\hbar^{2} Q^{2}}{2} \sum_{\lambda}\left(\hat{\mathbf{Q}} \cdot \mathbf{c}_{\nu}^{(\lambda)}\right)^{2} \hbar \omega_{\lambda} \operatorname{coth} y_{\lambda}\right\rangle_{\Omega} \\
& =\frac{\hbar^{2} Q^{2}}{2}\left\{\frac{1}{M} \hbar \omega_{T} \operatorname{coth} y_{T}+\frac{m_{2}}{m_{1} M}\left[\frac{2}{3} \hbar \omega_{L} \operatorname{coth} y_{L}+\frac{1}{3} \hbar \omega_{V} \operatorname{coth} y_{V}\right]\right\}
\end{aligned}
$$

Note that equations (14) - (16) have been used to derive the second equality (28). The orientational average in fact does not appear in (26) because of the result (24). The result for $\nu=1$ is shown in (28). The corresponding expression for $\nu=2$ follows directly from this. 
while

$$
c_{\nu}^{(T)}-c_{\nu^{\prime}}^{(T)}=0
$$

SO

$$
X_{12}=\exp \left[i Q d_{12} \mu\right] \exp \left[-\frac{\hbar^{2} Q^{2}}{2} \frac{M}{m_{1} m_{2}}\left(\left(1-\mu^{2}\right) \frac{\operatorname{coth} y_{L}}{2 \omega_{L}}+\mu^{2} \frac{\operatorname{coth} y_{V}}{2 \omega_{V}}\right)\right]
$$

The interference moments corresponding to(20) - (22) follow from this expression, but the resulting orientational averages in general can only be done numerically for the wide range of $Q$ that is involved. Note that for $S_{1}^{(12)}$ the expression becomes

$$
S_{1}^{(12)}=0
$$

identically by virtue of (24). Therefore the zeroth and first moments of this model scattering law are exactly as predicted by Placzek [2] for both self and distinct terms for all momentum and energy transfers. The results (26), (27) and (32) are completely general for an arbitrary number of modes.

\section{NUMERICAL COMPUTATIONS}

There are two aspects of the harmonic oscillator scattering law that require numerical computation to obtain the estimated differential cross section. These are the integration of the dynamic scattering law as given by equation (5) and the orientational averages given in equations (7). In addition it is necessary to estimate the modified Bessel functions to very high order and with large arguments. The latter is easily done by making using of the assympotic approximations, such as given in Abramovitz and Stegun [33], which when applied correctly give sufficiently accurate results. In practice the integral is quite straightforward since it becomes simply a sum over all permissable energy transfers along a path of constant TOF, but it can become time consuming with a large number of modes. 
The incident spectrum is defined using a standard formulation (see for example [35]) with the spectral parameters set for the particular neutron moderator applicable to the diffractometer in question. The incident spectrum is usually specified with respect to incident energy:

$$
\Phi\left(E_{i}\right)=\Phi_{\text {Max }}\left(E_{i}\right)+\Delta\left(E_{i}\right) \Phi_{\text {epi }}\left(E_{i}\right)
$$

where

$$
\begin{aligned}
\Phi_{\text {Max }}\left(E_{i}\right) & =J \frac{E_{i}}{T_{\text {mod }}^{2}} \exp \left(-\frac{E_{i}}{T_{\text {mod }}}\right) \\
\Phi_{\text {epi }}\left(E_{i}\right) & =\frac{\Phi_{0}}{E_{i}^{A}} \\
\Delta\left(E_{i}\right) & =\left[1+\exp \left(\frac{W_{1}}{\sqrt{E_{i}}}-W_{2}\right)\right]^{-1}
\end{aligned}
$$

with $J, T_{\text {mod }}, \Phi_{0}, A, W_{1}$ and $W_{2}$ parameters for the particular neutron moderator in question. The values used here were appropriate to the ISIS liquid methane moderator, which runs at approximately $100 \mathrm{~K}$, and were fit to an unpublished Monte Carlo estimate of the spectrum (Stuart Ansell, private communication). Specific values were $J=5.7, T_{\text {mod }}=0.011 \mathrm{eV}$, $\Phi_{0}=3.5, A=0.94, W_{1}=0.512 \mathrm{eV}^{\frac{1}{2}}$, and $W_{2}=1.5$. The absolute values and units of $J$ and $\Phi_{0}$ are not relevant here because these units are cancelled by the denominator in (5).

To convert to a spectrum per unit incident wavevector we apply the Jacobian:

$$
\Phi\left(k_{i}\right)=\Phi\left(E_{i}\right) \frac{\partial E_{i}}{\partial k_{i}}=2 \frac{E_{i}}{k_{i}} \Phi\left(E_{i}\right)
$$

with $E_{i}=\frac{\hbar^{2} k_{i}^{2}}{2 m}$ and $m$ is the mass of the neutron.

The detector efficiency can be approximated by a function of the form:

$$
D\left(k_{f}\right)=1-\exp \left(-\frac{\alpha}{\left(k_{f}\right)^{\beta}}\right)
$$

where $\alpha$ and $\beta$ are constants specific to a particular detector. Typically one would expect $\beta$ to be $\sim 1$, corresponding to a $1 / \mathrm{v}$ detector at high energy.

Finally the differential cross section, (7), has to be averaged over orientations, effectively averaging over $\mu$ in equations (15) and (16). This was achieved using 5 values of $\mu$ uniformly 


\section{SINGLE ATOM SCATTERING FOR A DIATOMIC MOLECULE CONTAIN- ING H AND D}

To illustrate the application of this method, a diatomic molecule consisting of masses $m_{1}=1$ and $m_{2}=17 \mathrm{amu}$ was setup with two sets of excitation energies. For Model (a) $\hbar \omega_{T}=20 \mathrm{meV}, \hbar \omega_{R}=200 \mathrm{meV}$, and $\hbar \omega_{R}=420 \mathrm{meV}$, and for Model (b) $\hbar \omega_{T}=15 \mathrm{meV}$, $\hbar \omega_{R}=60 \mathrm{meV}$, and $\hbar \omega_{R}=420 \mathrm{meV}$. Figure 2 compares the calculated differential cross sections over a range of scattering angles with measured data for water. The purpose of this figure is not to demonstrate agreement with the experimental data, which is generally not particularly good, but to show that changes to the vibrational frequencies in the model make a significant difference to the estimated differential cross section. In particular a model with vibrational excitations seems to be able to capture the underlying shape of the differential cross section - this cannot be achieved with simpler models for $S(Q, \epsilon)$ such as the ideal gas model. This hints that the use of vibrational excitations might be a tangible way for developing a robust correction for inelasticity effects in diffraction experiments involving hydrogen, but to achieve this, a model which involves more than three modes is almost certainly going to be needed. This will require development of a more sophisticated method of summing the different modes, as the length of the calculation increases exponentially with the number of modes.

Another observation is that the absolute levels of the measured data do not generally agree well with the levels predicted by the calculation, and there are quite marked variations from detector to detector. It is not obvious what might cause this variation in the level of the measured data. In the data analysis, corrections which multiply the data are the attenuation corrections, the normalisation to scattering cross section per atom, and the division by the data from a standard vanadium sample to put the data on an absolute cross section scale. Corrections which subtract from the data include backgrounds, empty container measurements, and multiple scattering contributions. The latter are particularly difficult to estimate since they involve neutron energy change each time a neutron is scattered with consequent change in cross-section. Hence it is possible the multiple scattering has been 


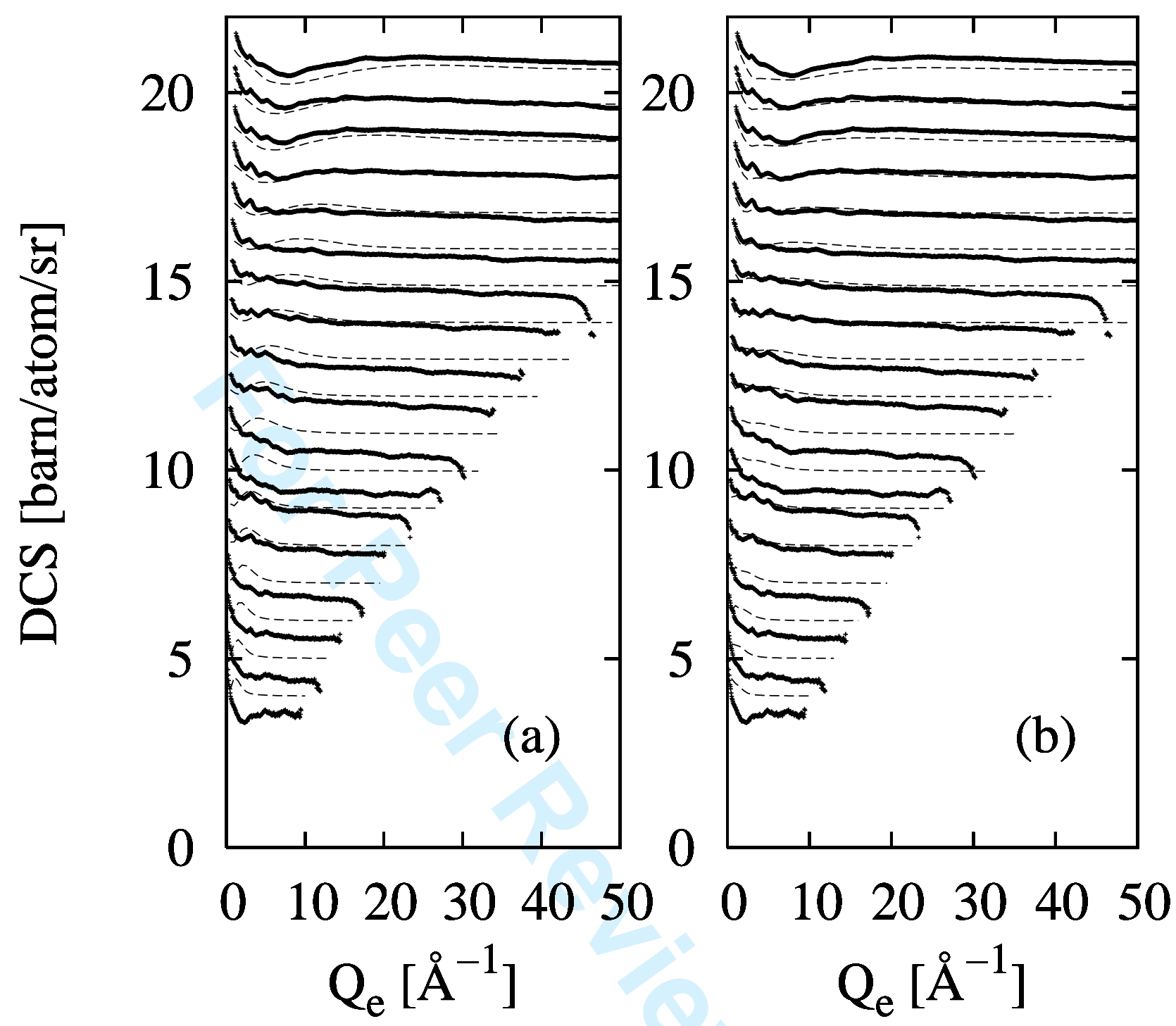

FIG. 2: Calculated (dashed lines) and experimental (dots) TOF differential cross sections (DCS) for light water, as obtained over a range of scattering angles from $3.8^{\circ}$ (bottom) to $35.4^{\circ}$ (top) on the SANDALS diffractometer at ISIS, as a function of elastic wave vector change $Q_{e}$. The left hand side corresponds to Model (a) and the right hand side corresponds to Model (b) as described in the text.

oversubtracted. This however does not explain the variation between detectors.

The general assumption is that the variations of level are additive rather than multiplicative, that is they do not affect the underlying interference scattering that is of interest for the structure of the fluid. Hence when all datasets from a range of scattering angles are merged to form a single diffraction pattern, the effects of inelasticity in the single atom 
scattering become smeared together. Merging is accomplished here by forming the arithmetic mean of the data from those detectors which contribute to a particular $Q_{e}$ value. This is demonstrated for models (a) and (b) in figure 3, where it can be seen that the detailed structure of individual detectors is smeared out when the detectors from a range of angles are merged. However direct merging of the experimental data cannot be undertaken in this manner because of the level shifts between detectors, as seem in Fig. 2. This is revealed in the small steps in the merged model calculations of Fig. 3. Hence it is necessary to subtract the single atom background, prior to merging the different detectors. A practical scheme for merging detectors in described in the next section, VID.

To demonstrate that the problems with self scattering are really only associated with light hydrogen Fig. 4 shows the same plot as Fig. 2 but for heavy water $\left(\mathrm{D}_{2} \mathrm{O}\right)$ instead of light water. The masses used here in the diatomic model for heavy water were $m_{1}=2$ and $m_{2}=18$ amu, with the vibrational frequencies derived from model (a) above, multiplying by the square root of the ratio of effective masses of each oscillator $\sqrt{\frac{m_{1}^{\left(H_{2} \mathrm{O}\right)} m_{2}^{\left(\mathrm{H}_{2} \mathrm{O}\right)}}{M^{\left(\mathrm{H}_{2} \mathrm{O}\right)}} \frac{M^{\left(D_{2} \mathrm{O}\right)}}{m_{1}^{\left(D_{2} \mathrm{O}\right)} m_{2}^{\left(D_{2} \mathrm{O}\right)}}}=$ 0.724, as would be appropriate for a harmonic oscillator. The modified frequencies are $\hbar \omega_{T}=14.5 \mathrm{meV}, \hbar \omega_{R}=145 \mathrm{meV}$, and $\hbar \omega_{R}=304.5 \mathrm{meV}$. Now we see that the interference signal is a much larger fraction of the differential scattering cross section and, although there are still some smaller discrepancies between data and model, the overlap between detector banks and model calculation is much more satisfactory. Hence the problem with removing inelasticity effects from diffraction data arises primarily for materials which contain light hydrogen: for any heavier atoms the problem of removing the inelastic single atom scattering is much smaller and can probably be dealt with using approximate methods.

It is worth emphasizing that the model being developed here is not intended to be an exact replica of the actual dynamical response of the material in question. Hence in the current example a linear diatomic molecule with three distinct normal modes has been used to represent a triatomic planar molecule with up to 9 distinct modes. It is possible this approximation is not sufficiently accurate for water or for more complex molecules - hence the discrepancies seen in Fig. 2. What is intended at this stage is to capture those primary dynamic features which give rise the substantial inelasticity effects that are observed in practice, and to this extent it appears to be successful, even though it could not in its present form be used to perform quantitative corrections from water data measured on a TOF diffractometer. It is fully anticipated the current model will be generalised to include 

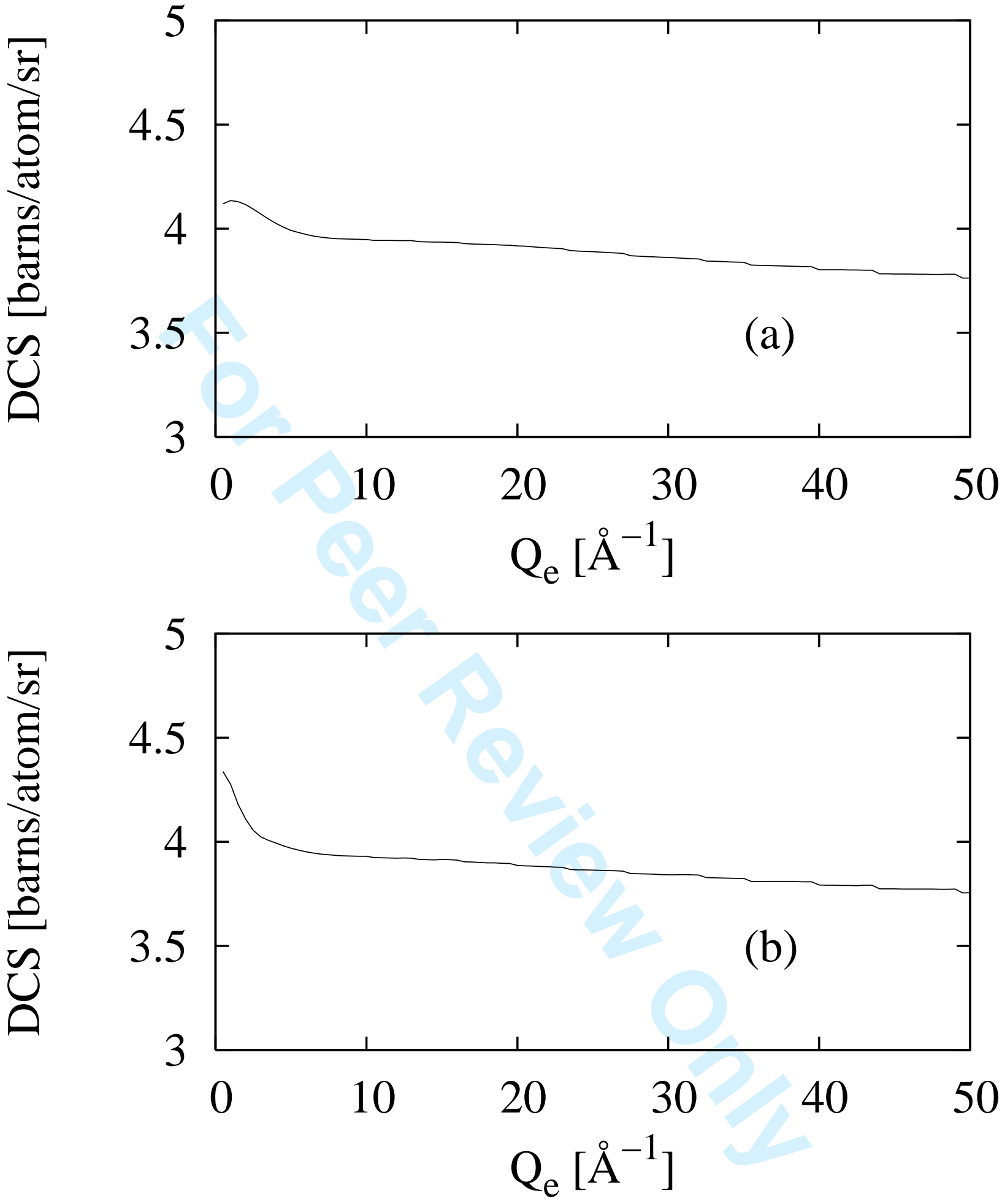

FIG. 3: Merged calculated differential cross sections for models (a) (bottom) and model (b) (top). Note the marked changes in this function with change of vibrational parameters. Small steps are visible at higher $Q_{e}$ due to the slightly different levels at the maximum $Q_{e}$ for each detector group. It is for this reason that direct merging of the diffraction data such as shown in Fig. 2 cannot be undertaken. 


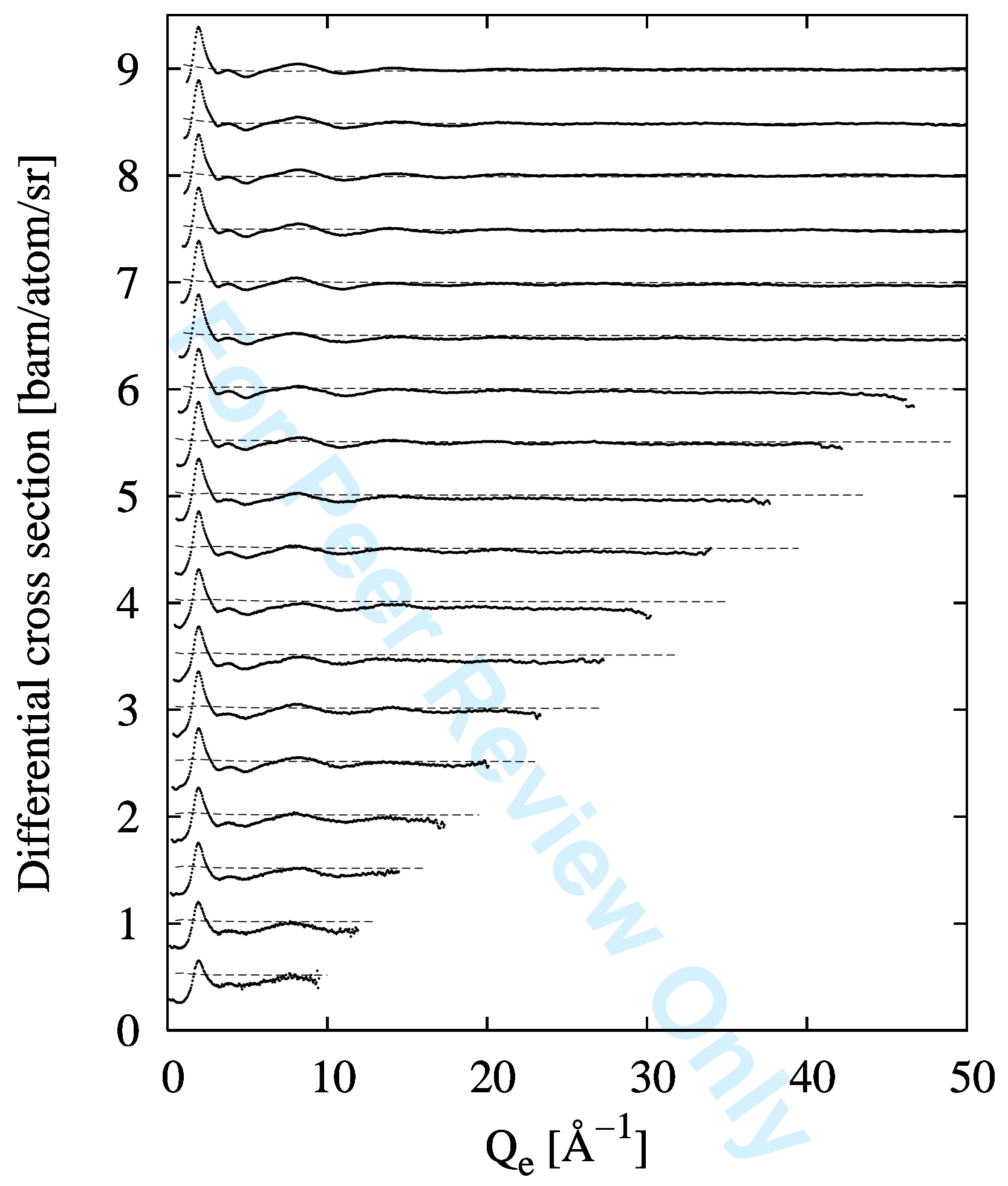

FIG. 4: Calculated (dashed lines) and experimental (dots) TOF differential cross sections (DCS) for heavy water $\left(\mathrm{D}_{2} \mathrm{O}\right)$, as obtained over a range of scattering angles from $3.8^{\circ}$ (bottom) to $35.4^{\circ}$ (top) on the SANDALS diffractometer at ISIS, as a function of elastic wave vector change $Q_{e}$. The dashed line corresponds to Model (a) with the frequencies modified according to the square root of the ratio of reduced mases, as described in section $\mathrm{V}$. 
these more complex geometries in the near future. Hence the present account is a "proof of principle" rather than a demonstration of the final product.

An additional factor is that the present account is treating primarily intra-molecular motions. However the use of vibrational excitations to represent translational and librational motions can be regarded as an attempt to include the inter-molecular forces that might be expected to operate on a molecule in the condensed state.

\section{THE "TOP HAT CONVOLUTION" (THC) METHOD FOR REMOVING THE SELF SCATTERING}

\section{A. Method}

Any practical scheme for removing backgrounds from diffraction data must be able to cope with a variety of backgrounds in diffraction data from a range of sources, including neutron and x-ray sources. In particular the background (single atom) scattering is often large and Q dependent, especially for light hydrogen with neutrons where the interference scattering may only be $5 \%$ or less of the self scattering - see Fig. 2 for example - or in x-rays at high $Q$ where the $Q$-dependent Compton scattering dominates. In addition, in order to separate the background scattering from the interference scattering, a criterion or set of criteria must be supplied in order to be able to say what is background and what is genuine interference scattering. There is no guarantee that such criteria can remove ALL the background, or that they will not remove some of the genuine interference scattering, but if they are physically based it means the background that is removed is defined by physical constraints rather than by the whim of the experimenter. The principles behind the proposed scheme here are:-

1) The background, although possibly dependent on $Q_{e}$, does not contain structural information in the form expected for the interference scattering, namely oscillations or peaks in $Q_{e}$-space corresponding to specific atom-atom correlations. In particular it will be assumed the $Q_{e}$-space background, whatever its cause, is made up mainly from low frequency components, which in turn would give rise to unphysical features at small $r$.

2 ) The background, when transformed to $r$-space, has the effect of introducing unphysical structure in the low- $r$ region of the corresponding radial distribution function, $g(r)$. This 
structure is manifest as peaks or dips in a region where normally $g(r)=0$, or, in the case of molecules, where $g(r)$ is known from molecular geometry.

Constraint (1) is hard to demonstrate in practice without a model for the dynamic scattering law. This was the reason for generating a model for the self scattering using the harmonic oscillator scattering law. Based on Fig. 3, it seems likely that the sort of inelastic interactions that arise from vibrational excitation, when smeared together over a range of scattering angles, will not give rise to periodic oscillations in the diffraction pattern that could otherwise be interpreted as specific atom-atom correlations. There might however be complications from the rise of the self scattering at low $Q_{e}$ which could potentially be construed as longer range structure in the material. Hence for materials containing light hydrogen, there will always be limitations to how accurately the small $Q$ scattering can be determined. If the rise in scattering at small $Q_{e}$ signal is strong, as might occur in a biphasic system for example, then the uncertainty from inelastic scattering will be small, but the method is otherwise not likely to be able to disentangle subtle long range structuring from the effects of inelasticity.

Constraint (2) is a restatement of ideas that have surfaced frequently over many years for removing unphysical effects from diffraction data - see for example the original work by Krogh-Moe and Norman [reference]. That is we know the behaviour of $g(r)$ at low $r$, so why not enforce it on our extracted structure factor (given that the data by themselves cannot do this)? Provided the constraints that have been applied are stated, then it is not unreasonable to use the modified diffraction data that emerge for a wider discussion of the structure of the material in question. After all, ANY form of data manipulation makes assumptions, many of which frequently go unstated.

Since the constraint (2) is to be applied in $r$-space the first task is to perform a Fourier transform of the data, $I\left(Q_{e}\right)$. (For simplicity we deal here with a monatomic material and neutron scattering, but the method is quite general for multicomponent materials and other radiation sources.) As has already been noted the $I\left(Q_{e}\right)$ consists of self and interference terms:

$$
I\left(Q_{e}\right)=I_{\text {self }}\left(Q_{e}\right)+I_{\text {int }}\left(Q_{e}\right)
$$

where 


$$
I_{\text {int }}\left(Q_{e}\right)=b^{2} 4 \pi \rho \int_{o}^{\infty} r^{2} h(r) \frac{\sin Q r}{Q r} d r
$$

with $g(r)=h(r)+1$ the radial distribution function being sought and $\rho$ the atomic number density of the material in question. (This ignores inelasticity effects in $I_{\text {int }}\left(Q_{e}\right)$, which will be discussed in a later section.) Generally $I_{\text {int }}\left(Q_{e}\right)$ will tail away to zero at high $Q_{e}$, but as shown in Fig. 2, $I_{\text {self }}\left(Q_{e}\right)$ will certainly not do this. Hence prior to inverting the Fourier transform, (40) it is necessary, in order to avoid large truncation effects, to form the difference function

$$
D\left(Q_{e}\right)=I\left(Q_{e}\right)-I_{\text {self }}\left(Q_{e}\right)
$$

As we have seen however, since we do not precisely know $I_{\text {self }}$, and since in any case the absolute normalisation on the diffraction data is known only to a certain accuracy, this subtraction cannot be done in general.

The idea behind the present method is to form a 'pseudo' self scattering function by convoluting the data with a "Top Hat" (TH) function in reciprocal space, and then use this convoluted data, $I^{\prime}\left(Q_{e}\right)$ as the self scattering background:

$$
I^{\prime}\left(Q_{e}\right)=\int_{R} I\left(\left|\mathbf{Q}_{e}-\mathbf{Q}_{e}^{\prime}\right|\right) T\left(\mathbf{Q}_{e}^{\prime}\right) d \mathbf{Q}_{e}^{\prime}
$$

where the integral proceeds over all of reciprocal space for which the data exist, and the top hat function $T\left(\mathbf{Q}_{e}\right)$ is given by:

$$
\begin{aligned}
T\left(\mathbf{Q}_{e}\right) & =\frac{3}{4 \pi Q_{T}^{3}}, & & \left|\mathbf{Q}_{e}\right| \leq Q_{T} \\
& =0, & & \left|\mathbf{Q}_{e}\right|>Q_{T}
\end{aligned}
$$

Given that the data are only specified at discrete values of $Q_{e}$, the Appendix shows how this convolution is performed in practice. In particular if $\Delta Q_{e}$ is the spacing between data points it will normally be arranged that $Q_{T} \leq n \Delta Q_{e}$ where $0<n \leq \sim 10$ so that the effect of the convolution is to smear $I\left(Q_{e}\right)$ only in the region near $Q_{e}$.

The aim therefore is to form the Fourier transform of the difference

$$
D^{\prime}\left(Q_{e}\right)=I\left(Q_{e}\right)-I^{\prime}\left(Q_{e}\right)
$$


namely:

$$
\left.d(r)=\frac{1}{(2 \pi)^{3} \rho} \int_{Q_{\min }}^{Q_{\max }} D^{\prime}\left(Q_{e}\right)\right) \exp \left[i \mathbf{Q}_{e} \cdot \mathbf{r}\right] d \mathbf{Q}_{e}
$$

where $Q_{\min }, Q_{\max }$ is the range of $Q_{e}$ values for which data are available. Making the use of the fact that the Fourier transform of a convolution is the product of the respective Fourier transforms, it is straightforward to show that

$$
d(r)=\left[G_{\text {self }}(r)+h_{\text {exp }}(r)\right]\left(1-f\left(Q_{T} r\right)\right)
$$

where

$$
\begin{aligned}
G_{\text {self }}(r) & =\frac{1}{2 \pi^{2} \rho r} \int_{Q_{\min }}^{Q_{\max }} Q_{e} I_{\text {self }}\left(Q_{e}\right) \sin \left(Q_{e} r\right) d Q_{e} \\
h_{\text {exp }}(r) & =\frac{1}{2 \pi^{2} \rho r} \int_{Q_{\min }}^{Q_{\max }} Q_{e} I_{\text {int }}\left(Q_{e}\right) \sin \left(Q_{e} r\right) d Q_{e}
\end{aligned}
$$

and

$$
f(x)=\frac{3}{x^{3}}[\sin x-x \cos x]
$$

The function $1-f(x)$ is shown in Fig. 5 . It is close to zero at low $x$, then rises close to unity for $x>\sim 4$. In other words it will heavily suppress low frequency structure in $Q$-space, but leave the higher frequency structure relatively intact. Precisely which frequency is cut off depends on the value of $Q_{T}$. For $x<<1 f(x) \approx x^{2} / 10$ so that for small enough $r$ and $Q_{T}$ the structure in $d(r)$ at a distance of say $r=r_{0}$ will be 4 times more suppressed than structure at $r=2 r_{0}$ and so on.

The significance of what has been achieved by using the smearing function (43) may not be apparent from this analysis. Suppose for example $I_{\text {self }}\left(Q_{e}\right)=C$, i.e. a constant as a function of $Q_{e}$, and we assume $Q_{\min } \approx 0$, then

$$
\begin{aligned}
G_{\text {self }}(r) & =\frac{C}{2 \pi^{2} \rho r} \int_{Q_{\min }}^{Q_{\max }} Q_{e} \sin \left(Q_{e} r\right) d Q_{e} \\
& \approx \frac{C}{2 \pi^{2} \rho r^{3}}\left[\sin \left(Q_{\max } r\right)-Q_{\max } r \cos \left(Q_{\max } r\right)\right]
\end{aligned}
$$




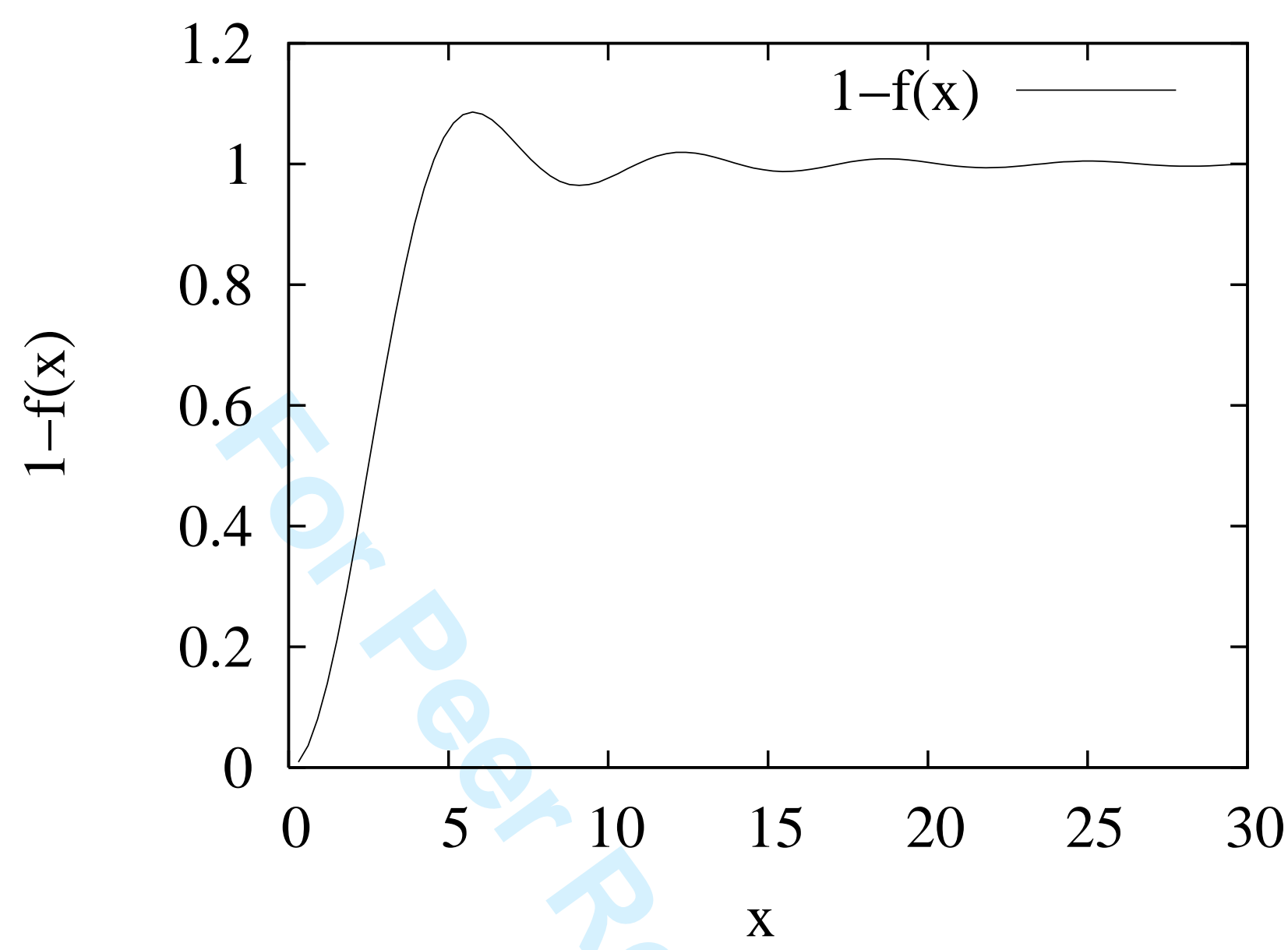

FIG. 5: The convolution function $1-f(x)$ which is defined in equation (49).

Figure 6 shows the effect of modifying $G_{\text {self }}(r)$ with $\left(1-f\left(Q_{T} r\right)\right)$ as given by (46), for $Q_{\max }=20 \AA^{-1}$ and $Q_{T}=0.3 \AA^{-1} . \quad G_{\text {self }}(r)$ shows the well known truncation oscillations as a function of $r$ and caused by the finite $Q_{\max }$ of the data. On the other hand in $G_{\text {self }}(r)\left(1-f\left(Q_{T} r\right)\right)$ these oscillations are barely visible. It is clear from this figure that, depending on the values of $Q_{\max }$ and $Q_{T}$, the smearing function suppresses the truncation oscillations drastically. Oscillations in $I\left(\mathbf{Q}_{e}\right)$ will be increasingly less suppressed as the frequency in $Q_{e}$ space increases.

Written in the form (46), it will be apparent that the convolution can easily be reversed in $r$ space because the result (45) simply needs to be divided by $\left(1-f\left(Q_{T} r\right)\right)$. By itself however this would achieve little since it would lead to the same function as would be derived by direct Fourier transform of the data. Instead the technique is to introduce contraint (2) at this point and assume that for some $r<r_{\min }$ we know $g(r)$ precisely, i.e. $g(r)=g_{0}(r)$ while for larger $r g(r)$ is derived by inverting the convolution in (46): 


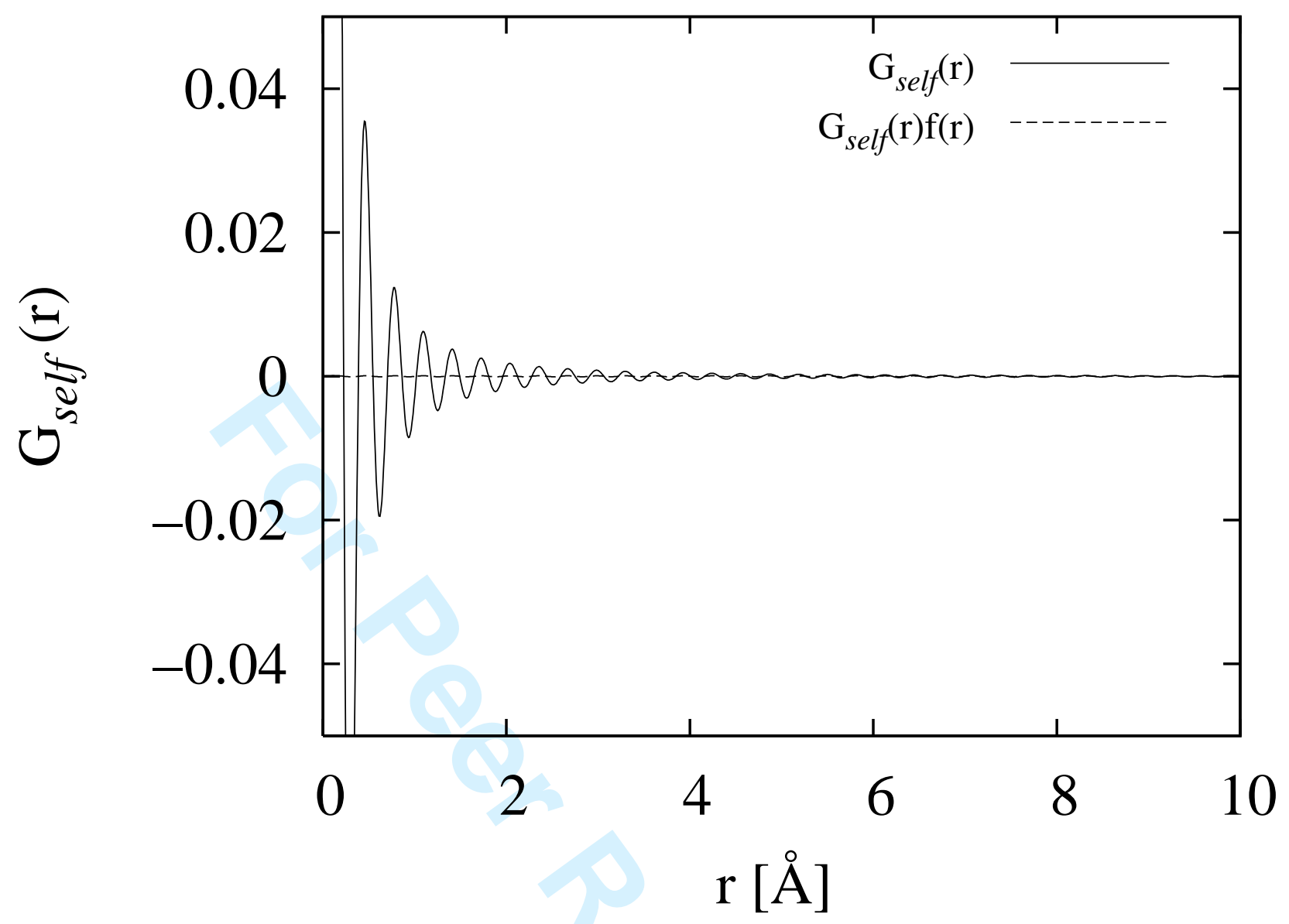

FIG. 6: The functions $G_{\text {self }}(r)$ (solid line) and $G_{\text {self }}(r)\left(1-f\left(Q_{T} r\right)\right)$ (dashed line) obtained from using $Q_{\max }=20 \AA^{-1}$ and $Q_{T}=0.3 \AA^{-1}$.

$$
\begin{aligned}
d_{\text {exp }}(r) & =g_{0}(r)-1, \quad r<r_{\min } \\
& =d(r) /\left(1-f\left(Q_{T} r\right)\right), \quad r \geq r_{\text {min }}
\end{aligned}
$$

with the assumption that $G_{\text {self }}(r)$ makes an insignificant contribution for $r \geq r_{\text {min }}$. If it does make a contribution in this region then the present method will not remove it, but it will remove it for $r<r_{\text {min }}$.

The object is to obtain an interference function in $Q_{e}$ space which has minimal contributions from the self scattering background and which satisfies our specified constraints. In principle one could simply Fourier transform (51) back to $Q_{e}$ space to achieve this, but if $g(r)$ is structured to high $r$ doing so might introduce further truncation effects. In addition unless the reverse transform is done carefully, this procedure will lose the statistical quality 
of the original data. A better plan is to generate an additional background function, $b(r)$, such that the function

$$
d_{\text {exp }}(r)=d(r)-b(r)
$$

This leads to

$$
\begin{aligned}
b(r) & =d(r)-g_{0}(r)+1, \quad r<r_{\min } \\
& =-d(r) f\left(Q_{T} r\right) /\left(1-f\left(Q_{T} r\right)\right), \quad r \geq r_{\min }
\end{aligned}
$$

Fourier transforming this to $Q_{e}$ leads to:

$$
B\left(Q_{e}\right)=4 \pi \rho \int r^{2} b(r) \frac{\sin Q_{e} r}{Q_{e} r}
$$

This extra background in $Q_{e}$ space is then subtracted from $D^{\prime}\left(Q_{e}\right)$ to yield an estimate of the interference differential cross section:

$$
I_{\text {int }}^{(e x p)}\left(Q_{e}\right)=I\left(Q_{e}\right)-I^{\prime}\left(Q_{e}\right)-B\left(Q_{e}\right)
$$

Because the function $f\left(Q_{T} r\right)$ is short ranged in $r$ the likelihood of truncation oscillations being transferred to $B\left(Q_{e}\right)$ is small. By this method the statistical quality of the original data is left intact, the specified constraints (1) and (2) are applied, and the effects of truncation in both $Q_{e}$ and $r$-spaces are held to a minimum, even when the data have a large self scattering component.

\section{B. Example of the Top Hat function method}

To illustrate the method, the structure factor, $H(Q)$, for a hard sphere fluid was generated within the Percus-Yevick approximation ([36], using a hard sphere diameter of $\sigma=2.8 \AA$ and a packing fraction $\eta=\pi \rho \sigma^{3} / 6=0.35$, which corresponds to an atomic number density of $\rho=0.030451 / \AA^{3}$. This structure factor is shown as the circles in figure 7 . To this data was added an artificial background of the form $2 \exp (-0.2 Q)$. The background was then estimated according to the methods of the previous section, using a minimum radius, $r_{\text {min }}=2.8 \AA$, to give the corrected $H(Q)$ (solid line in Fig. 7). 


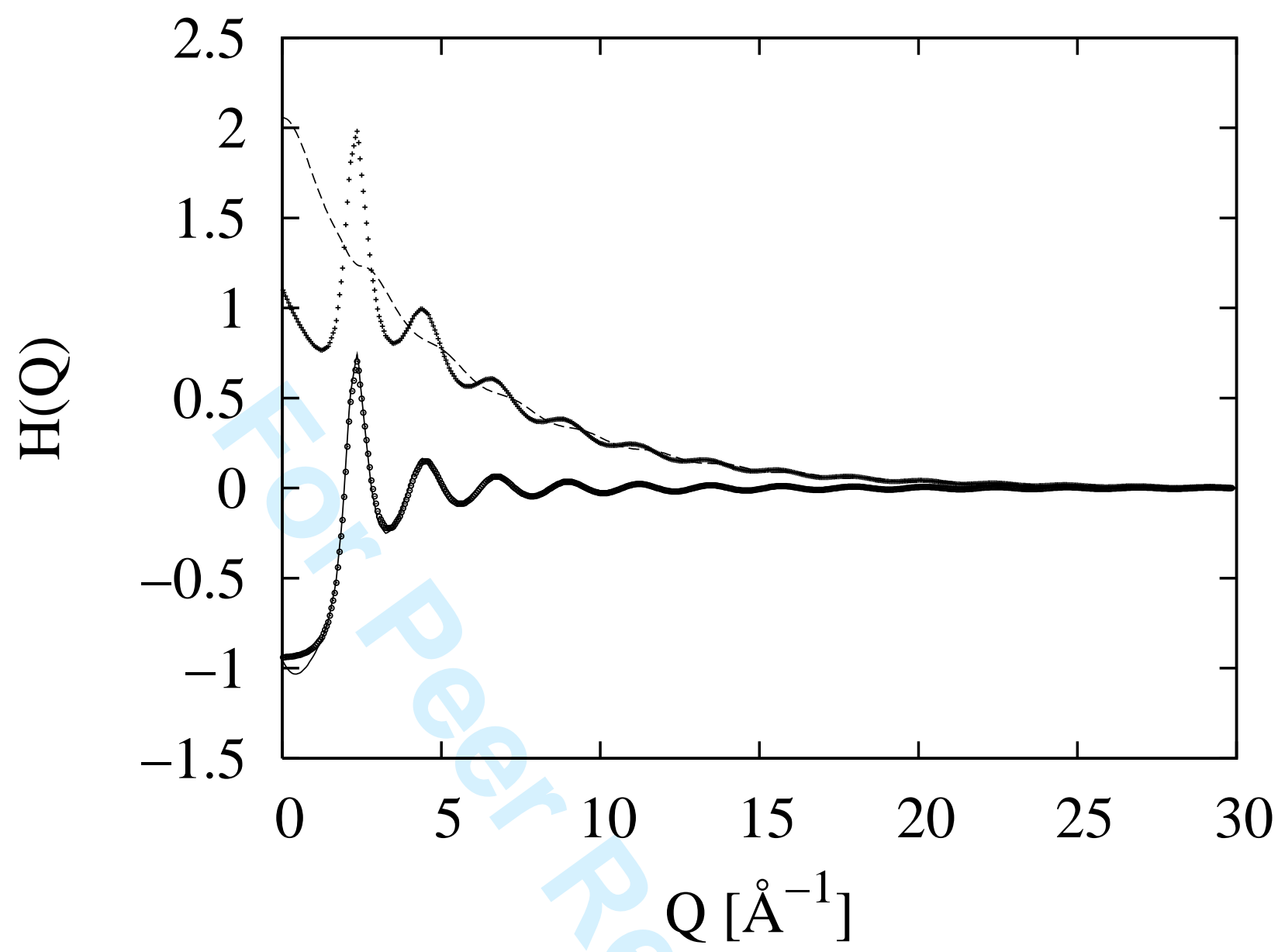

FIG. 7: Illustration of the "top hat" method of subtracting backgrounds from diffraction data. The circles show the Percus-Yevick hard sphere structure factor at a packing faction $\eta=0.35$, with $\sigma=2.8 \AA$. The crosses show the same data after adding a large $Q$-dependent background. The dashed line shows the background as estimated by the top hat method, and the solid line shows the reconstructed structure factor.

Fig. 8 compares the before and after corrected $h(r)$. A Lorch function [37] was applied to the data, with $Q_{\max }=30 \AA^{-1}$, to effect this transformation, because the oscillations in $h(Q)$ for a hard sphere fluid proceed to infinite $Q$ and so would otherwise give rise to significant truncation effects in the reverse Fourier transform. It can be seen that the effect of the large $Q$-dependent background has largely been ameliorated in the extracted radial distribution. Both original and corrected $h(r)$ functions also compare well with what would have been obtained if data to very large $Q$ were available (dashed line). 


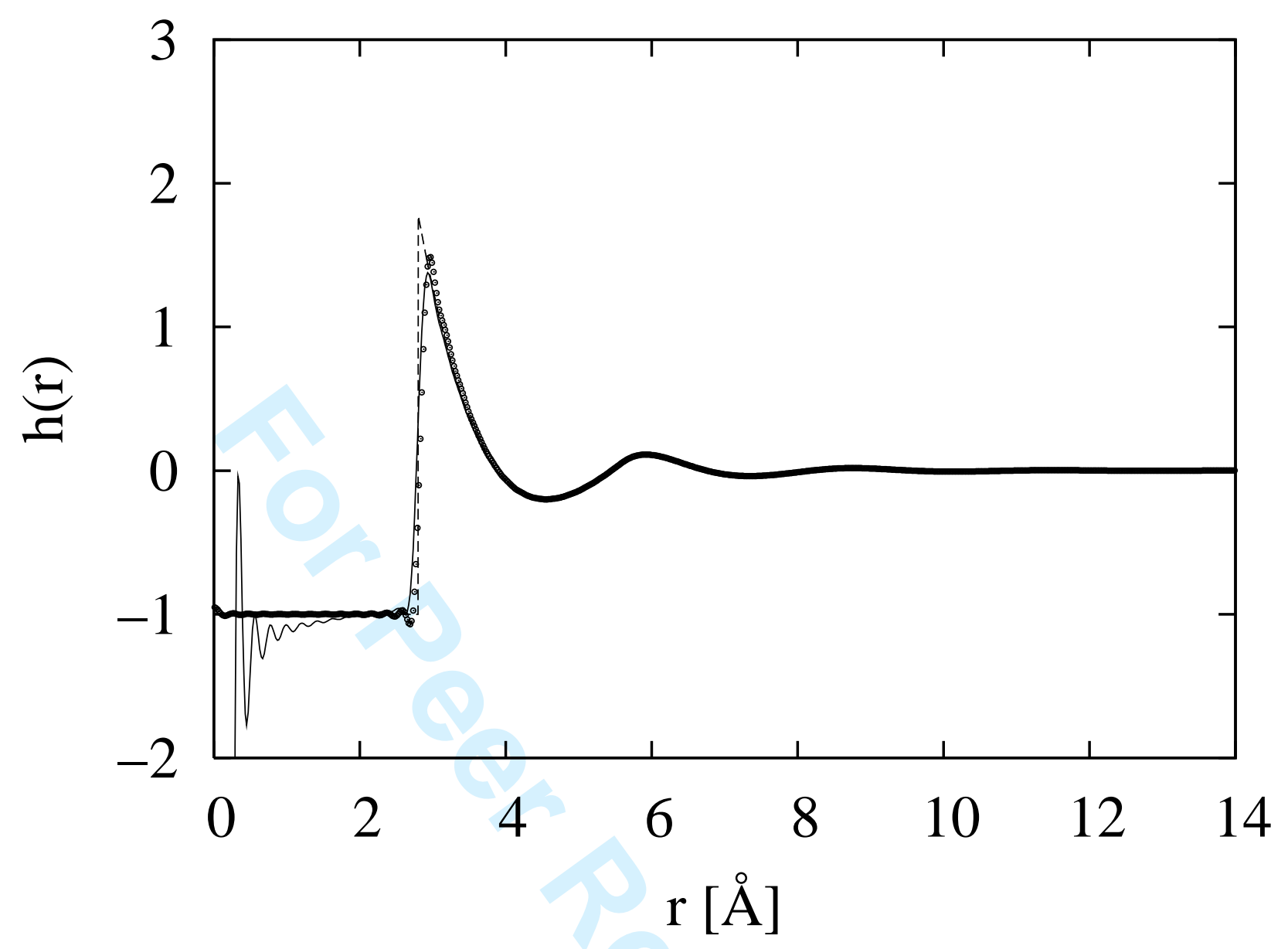

FIG. 8: Illustration of the "top hat" method of subtracting backgrounds from diffraction data. The circles show the result of direct Fourier transform of the original data, using a $Q_{\max }=30 \AA^{-1}$, compared with that obtained from the background corrected data (solid line). Also shown is the result of an "exact" transform of the original structure factor out to $Q_{\max }=500 \AA^{-1}$ (dashed line).

\section{Discussion of the "top hat" method}

Important aspects of the method to consider are the nature of the broadening function, equation (43), and the fact that the convolution is performed in 3-D rather than 1-D. Strictly, using any broadening function to smooth at set of data means that the useful range of the data is reduced. In the present instance for example, if the supplied data range from $Q_{\min }<$ $Q<Q_{\max }$, then the range of the smeared data is reduced to $Q_{\min }+Q_{T}<Q<Q_{\max }-Q_{T}$, since outside this range the smeared data are undefined. Hence it is imperative that the edge of the smearing function is sharp and that $Q_{T}$ is kept as small as is practical. The convolution is performed in 3-D so that the deconvolution and application of the boundary 


\section{Towards a practical scheme for merging data from multiple detector banks}

A conclusion from section V - see Fig. 2 - was that data measured at the same $Q_{e}$ value but at different scattering angles would not necessarily overlap, due to the different inelasticity effects at different angles. Hence direct averaging of the data from different detectors for each $Q_{e}$ is not possible in general. The top hat convolution method of section VI offers the possibility of performing this merge in a manner which does introduce the steps seen in Fig. 3. In essence, assuming the data for each detector occur over a significant $Q_{e}$ range, the top hat convolution and subtraction, equation (42) are performed on the data from each detector. The resulting difference data are then merged by forming the arithmetic mean of the data from all detectors that contribute to a particular $Q_{e}$ value. This leads to a single function, $D^{\prime}\left(Q_{e}\right)$ (equation (44)) which is the merging of all the data from all the detectors. This function is then used as input to the second part of the top hat convolution method, equations (45) - (56). In this way if there are inelastic features in the differential cross sections from individual detectors they will not create steps in the merged data, but will become smeared out in the averaging procedure, so reducing the possibility of them contributing to the final interference scattering.

\section{EFFECT OF INELASTICITY ON THE INTERFERENCE SCATTERING}

Previous sections have shown us that for light atoms, particularly $\mathrm{H}$, there may be significant effects on the single atom scattering due to inelasticity and that in general these effects cannot be calculated with sufficient quantitative accuracy to enable a direct subtraction of the self scattering. A realistic model of the scattering law suggests that inelasticity effects 
are unlikely to introduce an oscillatory function that would give rise to specific peaks in $r$-space - see Fig. 3 for example. Instead an empirical method was introduced using top hat convolution which appeared to be able to make an approximate subtraction of any large $Q_{e}$ dependence in the single atom scattering. It remains to be established whether the resulting interference function is likely to be affected by inelastic effects in the interference scattering.

The fact that the first moment of the dynamic scattering law for intereference scattering is zero - [2] and equation (32) - cannot be taken to imply there is no energy transfer associated with interference scattering. The first moment goes to zero in the harmonic oscillator scattering law because the arguments to the modifed Bessel functions in equation (8) are negative for the librational and vibrational modes of the diatomic model as a result of the definition of the normal modes (13). This leads to negative intensity for odd values of $n_{\lambda}$.

On the other hand the product $\left(\mathbf{Q} \cdot \mathbf{c}_{\nu}^{(\lambda)}\right)\left(\mathbf{Q} \cdot \mathbf{c}_{\nu^{\prime}}^{(\lambda)}\right) \sim \frac{1}{M}$ in $(8)$, so that the same argument is correspondingly much smaller compared to the self scattering from $\mathrm{H}$ or $\mathrm{D}$. This means inelastic scattering is much more likely to be concentrated near $\omega=0$ than near the recoil energy of the proton.

In order to evaluate the effect of inelasticity on the interference scattering, the parameters of model (a) as used in section $\mathrm{V}$ were used to calculate the interference differential cross section for the $\mathrm{OH}$ bond in $\mathrm{H}_{2} \mathrm{O}$ and $\mathrm{D}_{2} \mathrm{O}$. In addition a second model with equal masses of $m_{1}=m_{2}=2 \mathrm{amu}$ and the same frequencies as model (a) for $\mathrm{D}_{2} \mathrm{O}$ was used to estimate the $\mathrm{HH}$ intramolecular interference function for both $\mathrm{H}_{2} \mathrm{O}$ and $\mathrm{D}_{2} \mathrm{O}$. Assumed bond lengths were $0.98 \AA$ for $\mathrm{OH}$ and $\mathrm{OD}$ and $1.55 \AA$ for $\mathrm{HH}$ and $\mathrm{DD}$ distances.

The estimated $\mathrm{OH}$ and $\mathrm{OD}$ intramolecular structure factors for $\mathrm{H}_{2} \mathrm{O}$ and $\mathrm{D}_{2} \mathrm{O}$ are shown in Figure 9 and compared with what would be predicted for the same bonds in the absence of inelasticity effects. The ideal distinct scattering for this case is given by

$$
F\left(Q_{e}, d_{O H}\right)=\frac{\sin Q_{e} d_{O H}}{Q_{e} d_{O H}} \exp \left(-a_{O H} Q_{e}^{2}\right)
$$

where $a_{O H}$ is the Debye-Waller factor, and $d_{O H}$ is the $\mathrm{OH}$ bond length. In the present instance, $a_{O H}$ was chosen to give the best agreement with the harmonic oscillator calculation, and $d_{O H}$ was set to the same as the calculation, namely $0.98 \AA$. A small shift to lower $Q_{e}$ in the harmonic oscillator calculation is observed compared to the expected function, suggesting that the primary effect of inelasticity is to make the bond length appear slightly longer. Note that this effect is apparent in both $\mathrm{OH}$ and $\mathrm{OD}$ calculations. To estimate the size 


\section{INELASTICITY CORRECTIONS AT A REACTOR (FIXED WAVE- LENGTH) NEUTRON SOURCE}

When the incident wavelength is fixed by a monochromator, as in a reactor neutron source experiment, then the elastic and incident wavevectors become the same, $k_{e}=k_{i}$, and the final wavevector ranges over all values, $k_{f} \geq 0$. Theoretically these conditions can be obtained by setting the flight path ratio $R=0$ in the TOF equation (3) and the Jacobian (6). Hence the revised integral (5) becomes:

$$
\frac{d \sigma}{d \Omega}=b^{2} \int_{\text {const. } \theta} \frac{D\left(k_{f}\right)}{D\left(k_{i}\right)} \frac{k_{f}}{k_{i}} S(Q, \epsilon) d \epsilon
$$

Because the incident energy is fixed the possibility for sampling a large number of vibrational excitations is significantly reduced, so the calculation proceeds much more rapidly. Otherwise however the calculation is identical to the TOF case.

Figure (12) shows the consequence of running this calculation for the same harmonic 

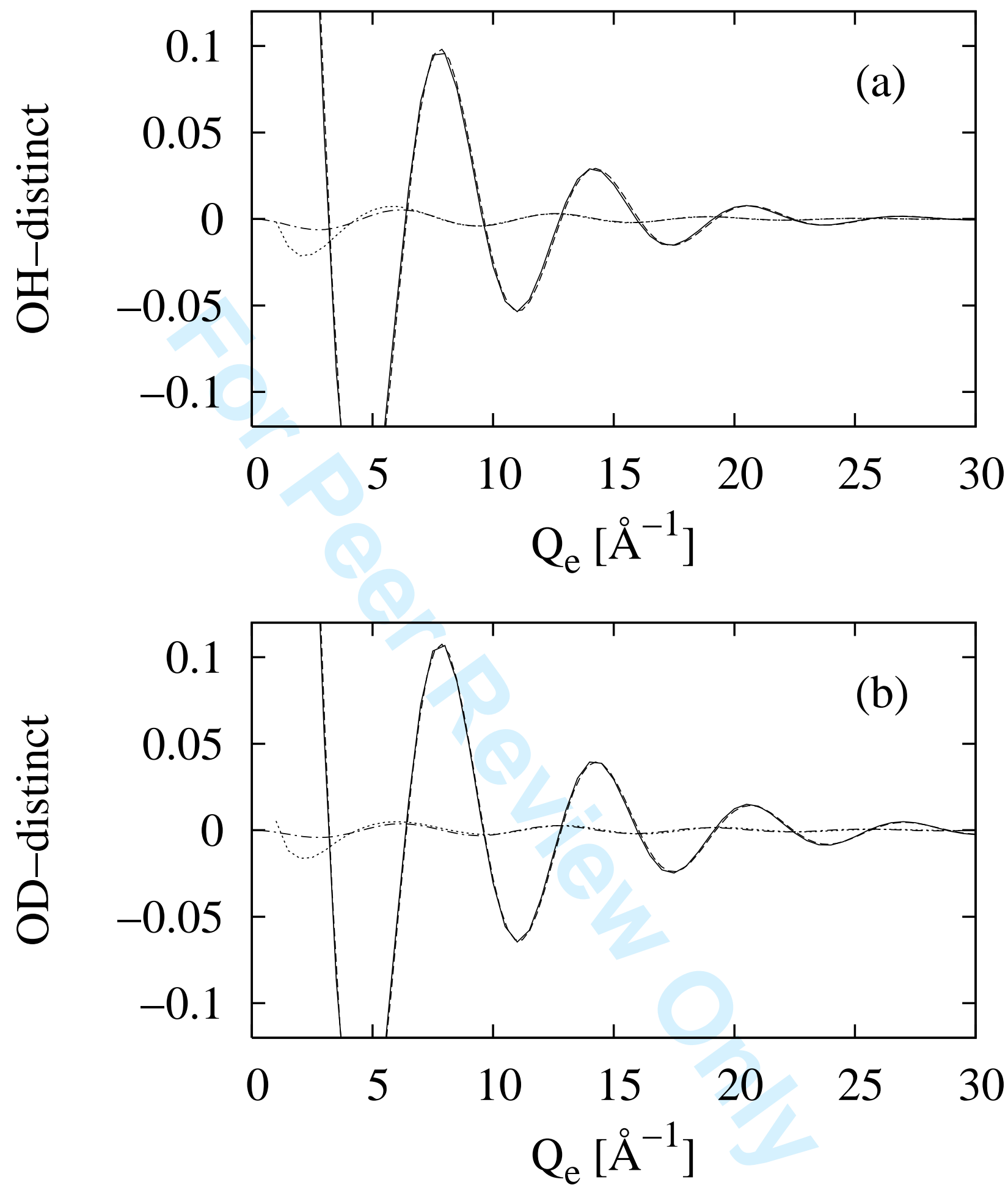

FIG. 9: $\mathrm{OH}$ and $\mathrm{OD}$ intramolecular structure factor for $\mathrm{H}_{2} \mathrm{O}$ (a) and $\mathrm{D}-2 \mathrm{O}$ (b) as estimated from the harmonic oscillator model (solid line) for a scattering angle of $35.4^{\circ}$. Also shown is the (expected) function, equation (57) (dashed line). The difference functions $F\left(Q_{e}, d_{O H}\right)-F\left(Q_{e}, d_{O H}^{\prime}\right)$ are shown in this figure as dot-dash lines, and compared with the difference $I_{O H}\left(Q_{e}\right)-F\left(Q_{e}, d_{O H}\right)$ (dotted line). For (a) $\left(\mathrm{H}_{2} \mathrm{O}\right) d_{O H}^{\prime}=0.986 \AA$, while for $(\mathrm{b})\left(\mathrm{D}_{2} \mathrm{O}\right) d_{O H}^{\prime}=0.984 \AA$. 


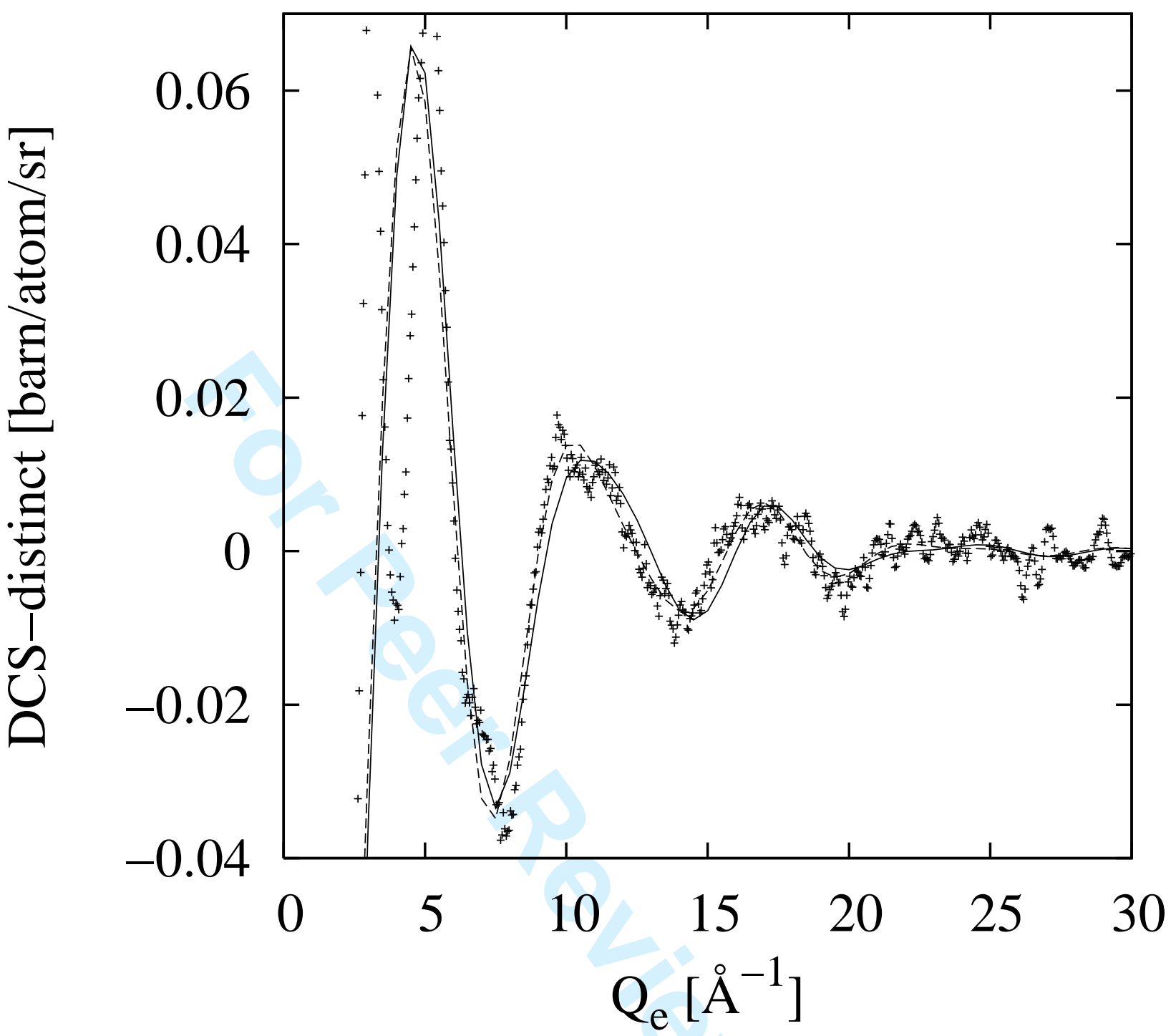

FIG. 10: Interference differential scattering cross section for $\mathrm{H}_{2} \mathrm{O}$. This function includes both $\mathrm{OH}$ and $\mathrm{HH}$ interference functions estimated from the harmonic oscillator model and combined according to their neutron weights. The crosses show the data after merging 18 detector angles, using the top hat convolution method described in section VI. The solid line shows the estimated function, assuming an $\mathrm{OH}$ bond length of $0.98 \AA$. The dashed line shows the estimated function assuming an $\mathrm{OH}$ bond length of $1.01 \AA$.

oscillator model as previously, using the masses and excitation energies given in the figure caption. Also shown are the cases where the rotational excitation energies are varied $\pm 20 \mathrm{meV}$. Not only does this set of parameters produce an excellent fit to the data, it is seen that the fit can be controlled quite sensitively by the choice of vibrational energies. 


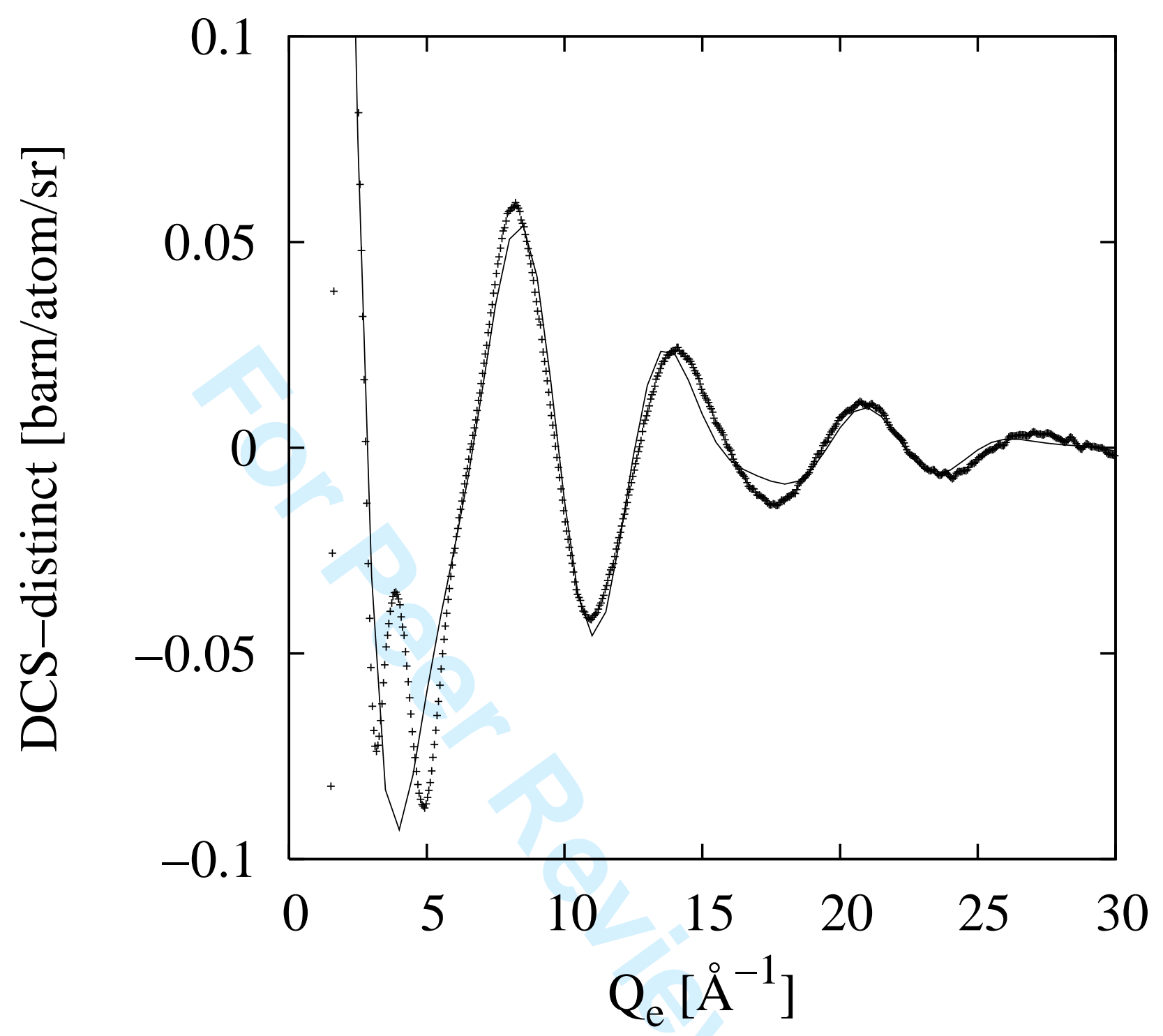

FIG. 11: Interference differential scattering cross section for $\mathrm{D}_{2} \mathrm{O}$. This function includes both $\mathrm{OH}$ and $\mathrm{HH}$ interference functions estimated from the harmonic oscillator model and combined according to their neutron weights. The crosses show the data after merging 18 detector angles, using the top hat convolution method described in section VI. The solid line shows the estimated function, assuming an $\mathrm{OH}$ bond length of $0.98 \AA$.

Hence there is here the possibility of parameterising the single atom scattering when light hydrogen is present in terms of vibrational excitation energies.

Figure (13) repeats this calculation for heavy water, and once again the same good agreement with the experiment is found. Finally figure (14) shows the calculated $\mathrm{OH}$ interference scattering for $\mathrm{D}_{2} \mathrm{O}$, and compares it to what would be measured if there were no inelasticity 


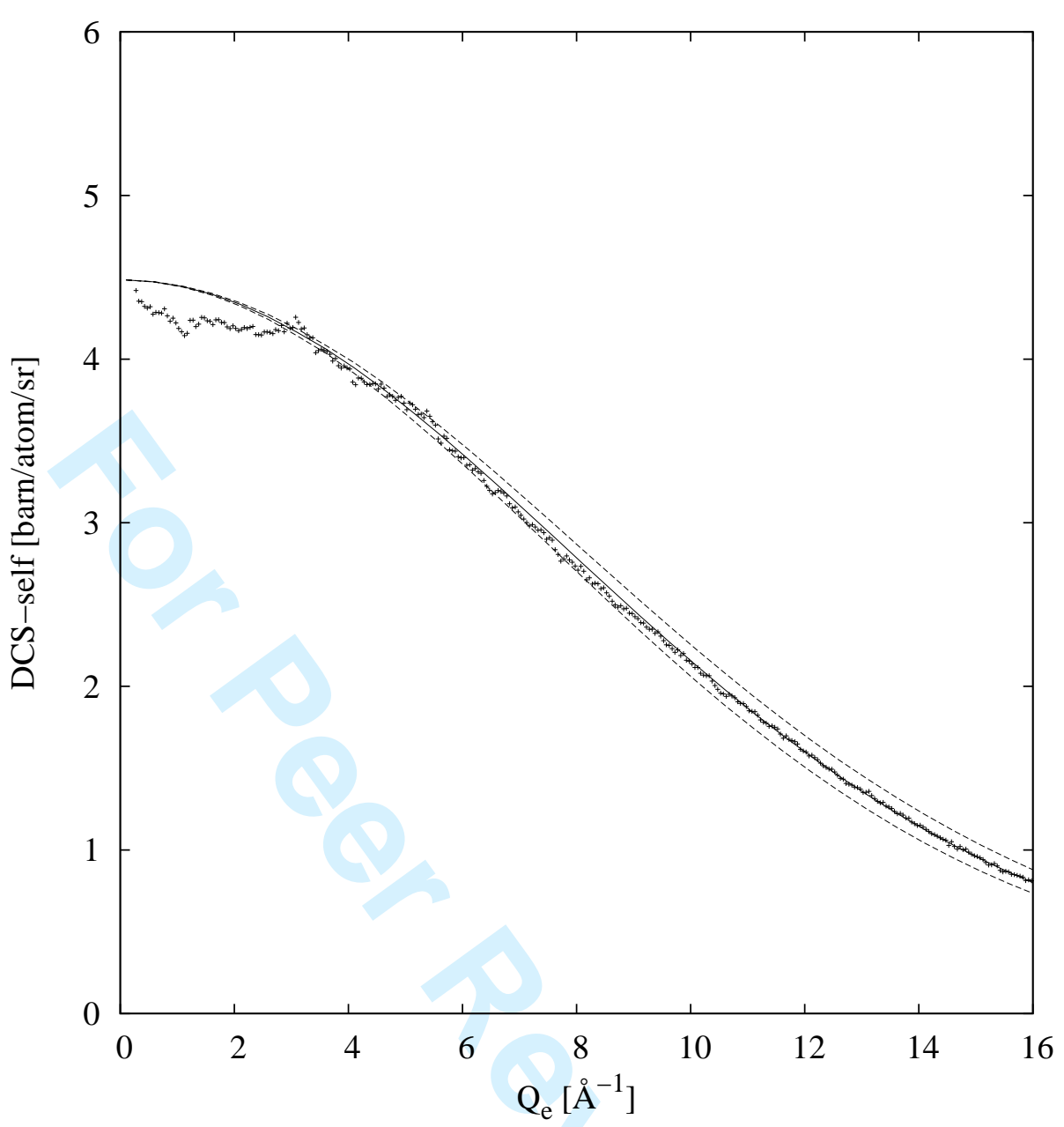

FIG. 12: Predicted self differential scattering cross section for $\mathrm{H}_{2} \mathrm{O}$ (solid line) obtained using the harmonic oscillator scattering law for a diatomic molecule, with masses $m_{H}=1, m_{O}=17$, the translational, rotational and vibrational excitation energies set to 23,230 and $420 \mathrm{meV}$ respectively, and the incident wavelength set to $0.7 \AA$. Also shown as dashed lines are the cases where these excitation energies 21, 210 and 420meV (lower curve) and 25, 250 and $420 \mathrm{meV}$ (upper). The crosses show the data for light water as measured on D4C.

correction. It will be noted the oscillations in the calculated functions have a slightly longer period in $Q$ space than the static (zero inelasticity) function (dashed line), implying that the effect of inelasticity in the reactor experiment is to make bond lengths appear slightly shorter than their actual values. Note that this is the exact reverse to what was observed in the TOF diffraction experiment. Moreover the effect of inelasticity on the interference diffraction pattern appears to get progressively worse with increasing $Q$ (which means in- 


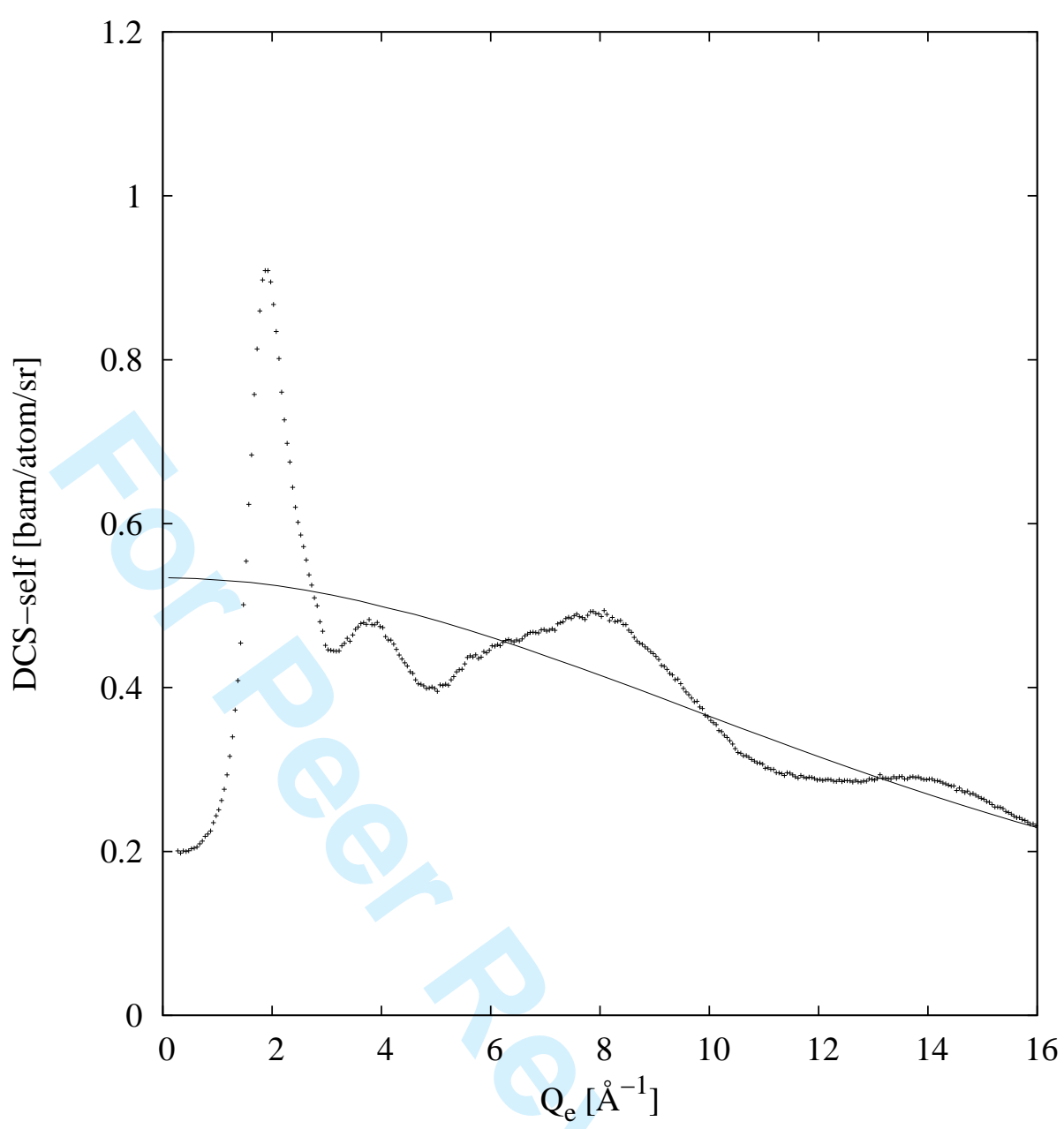

FIG. 13: Predicted self differential scattering cross section for $\mathrm{D}_{2} \mathrm{O}$ (solid line) using masses of 2 and 18 for $\mathrm{D}$ and $\mathrm{O}$ respectively, and vibrational energies of $14.5,145$ and $304.5 \mathrm{meV}$ respectively. The crosses show the data for heavy water as measured on $\mathrm{D} 4 \mathrm{C}$

creasing scattering angle, since the wavelength is fixed). This tendency is consistent with that predicted by Powles [7] using an expansion of the dynamic scattering law similar to that proposed by Placzek [2].

\section{DISCUSSION AND CONCLUSIONS}

The foregoing account has explored the ramifications of using an harmonic oscillator model for the dynamic scattering law to estimate the effects of inelasticity on diffraction experiments from materials containing light atoms, particularly hydrogen. It is difficult to 


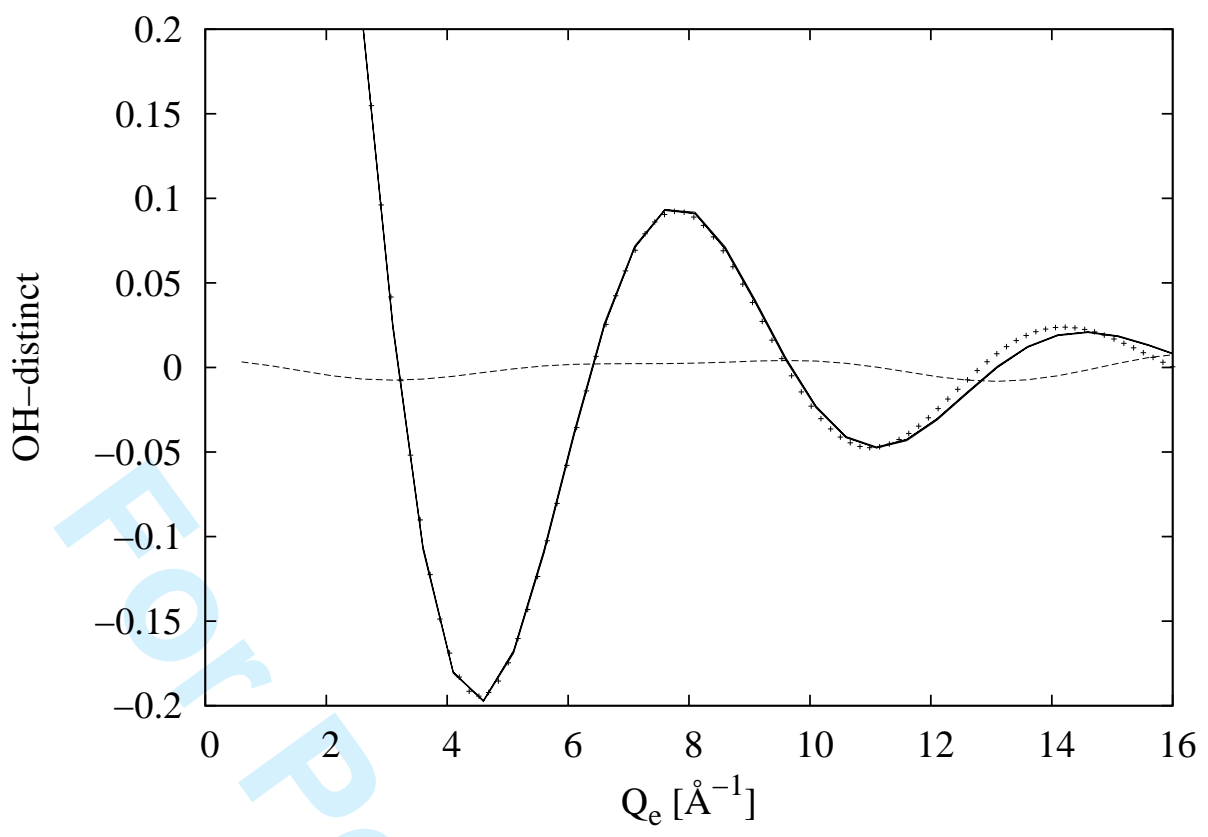

FIG. 14: $\mathrm{OH}$ interference differential scattering cross section for $\mathrm{D}_{2} \mathrm{O}$ calculated for $\mathrm{D} 4 \mathrm{C}$, estimated from the harmonic oscillator model with excitation energies given in the caption to Fig. (13). The solid line shows the estimated function, assuming an $\mathrm{OH}$ bond length of $0.98 \AA$, while the crosses show the function that would be obtained if there were no inelasticity. The dashed line shows the difference between these two. The conclusion is that inelasticity at a reactor source tends to make the bond length appear shorter than it actually is.

compare these results with previous work as there are relatively few previous attempts to calculate these effects from hydrogen in detail. The early work using models $[12,16,19]$ really only applied to low energy fixed wavelength experiments and did not address the question of inelasticity in the interference scattering. A later attempt with a different model, [39] was not overwhelmingly successful in the high energy limit, [32, 40]. Currently all the known methods of removing inelasticity effects from diffraction data containing hydrogen rely on some form of background subtraction using polynomials in $Q$ or an equivalent procedure such as the top hat convolution procedure described in section VI above. Even in the present work, the harmonic oscillator diatomic model does not fully explain the observed $Q$ dependence of the time-of-flight diffraction patterns for light water, Fig. 3, but is indicative that a suitable model might be found using this formalism. For reactor diffraction on the other hand the present formalism appears to give an accurate inelasticity correction. The 
difficulty with any form of model scattering law that is invoked, apart from the obvious points that it must be applicable over a huge range of energy transfers, from sub-meV to several tens of $\mathrm{eV}$, and contain known physics such as the correct first moment and the detailed balance condition, is obtaining reliable parameters for the model. In this case it may be that deep inelastic neutron scattering (DINS) [32] can provide us with the high momentum recoil profile that any model for the scattering law must satisfy.

For interference scattering, we appear to be on safer ground, since as pointed out by Powles [9] the effective mass involved is greater (see section III A above) than for the self scattering, so inelasticity effects are likely to intrinsically smaller. This was indeed demonstrated in sections VII and VIII above, where for TOF the effect was less than $1 \%$ in the bond length provided the scattering angle is less than $\sim 40^{\circ}$, while for the reactor case it appears to increase with increasing $Q$. Powles [8] observed that for the reactor experiment the oscillations have a slightly longer period as a result of inelasticity than would be observed without inelasticity, and the present results are in agreement with this, Fig. 14. In the case of the TOF experiment, Powles predicts [9] the effect of inelasticity is to also increase the period of the oscillations with $Q$ compared to the expected response without inelasticity, whereas in section VII above the opposite behaviour is observed, namely the interference oscillations with inelasticity have a slightly shorter period than expected, Fig. 9. However the two examples are not comparable. Powles [9] shows the case of a scattering angle of $150^{\circ}$, whereas the data shown here correspond to scattering angles $\leq 40^{\circ}$. Powles also shows that the effect will diminish rapidly at low scattering angles, so it may be the present observations with inelasticity are the same as those without inelasticity within the calculational error.

Clearly the use of a diatomic molecule to represent the dynamics of something more complicated such as water is not an adequate representation. However simply extending the method to more a realistic number vibrational modes will cause a rapid increase in computing time due to the nature of the product (which is in effect a convolution) in equation 7 . Hence as the number of modes required at high momentum and energy transfers increases some approximation for simplifying the lower frequency modes, such as the impulse approximation [32], is needed. It is hoped to report on progress in this direction in a future publication.

Overall therefore the present results are encouraging, and suggest that the time when we can achieve accurate inelasticity corrections for light atoms is not far away. 


\section{Appendix: Performing the top hat convolution on discrete data}

Experimental data represent continuous functions, but of necessity have to be measured at discrete intervals in the relevant quantity - $Q$ in the present instance. In essence the value of the intensity, $I(Q)$ is averaged over some interval $\Delta$ around each specified $Q$ value, and assumed to be uniform within that interval, $Q-\frac{1}{2} \Delta<Q<Q+\frac{1}{2} \Delta$. In the present instance $Q$ extends from 0 to $\infty$, and is strictly a vector, $\mathbf{Q}$, except that for fluids and glasses $I(\mathbf{Q})$ is isotropic with respect to the directions of $\mathbf{Q}$, so the 3-dimensional character of the intensity is often ignored. But it cannot be ignored when performing convolutions such as equation (42).

It is convenient to divide $Q$ into a series of bins: $Q_{m}=m \Delta$ with $m=0,1,2, \ldots M$. The bin boundaries will go $0, \frac{1}{2} \Delta, \frac{3}{2} \Delta, \ldots, \frac{2 m+1}{2} \Delta$, etc. Equally the top hat function, equation (43), is specified at intervals $Q_{j}=j \Delta$ with $j=0,1,2, \ldots n$ and is uniform over this range of intervals, with $Q_{T}=\left(n+\frac{1}{2}\right) \Delta$.

With these assumptions it is possible to replace the 3-D convolution of equation (42) which would be very time consuming - with a 1-D convolution. The problem is to distribute the intensity at each $Q=Q_{m}$ uniformly over a sphere of radius $Q_{T}$, then re-sum all the contributions for a given $Q$. To do this it is necessary calculate the volume of a spherical shell of radius $Q_{l}=l \Delta$ and thickness $\Delta$ which intersects another sphere (the top hat function) of radius $Q_{T}$ placed at $Q=Q_{m}$. This volume will only exist if $\max [0, m-n] \leq l \leq m+n$. First it will be noted that the surface area of intersection of a sphere of radius $Q$ with a sphere of radius $Q_{T}$ at position $Q=Q_{m}$ is given by:

$$
\begin{aligned}
A\left(Q, Q_{m}\right) & =2 \pi\left[Q^{2}-\frac{Q^{3}+Q\left(Q_{m}^{2}-Q_{T}^{2}\right)}{2 Q_{m}}\right], \quad\left|Q_{m}-Q_{T}\right|<Q \leq Q_{m}+Q_{T} \\
& =4 \pi Q^{2}, \quad 0 \leq Q \leq\left|Q_{m}-Q_{T}\right| \\
& =0 \quad \text { otherwise. }
\end{aligned}
$$

This is then integrated in the interval $Q_{l}-\frac{1}{2} \Delta<Q<Q_{l}+\frac{1}{2} \Delta$ and normalised to the volume of the top hat function sphere, $\frac{3}{4 \pi Q_{T}^{3}}$, as in (43). The result is: 


$$
\begin{aligned}
V\left(Q_{l}, Q_{m}\right) & =\frac{3}{4 \pi Q_{T}^{3}} \int_{Q_{l}-\frac{1}{2} \Delta}^{Q_{l}+\frac{1}{2} \Delta} A\left(Q, Q_{m}\right) d Q \\
& =\frac{1}{(2 n+1)^{3}}\left\{12 l^{2}+1-\frac{3}{2 m} l\left[4 l^{2}+4 m^{2}-(2 n+1)^{2}+1\right]\right\}, \\
& =\frac{2-\delta(m)}{(2 n+1)^{3}}\left\{12 l^{2}+1\right\}, \quad 0 \leq l \leq|m-n|
\end{aligned}
$$

where $\delta(l)=1$ if $l=0$ and $\delta(l)=0$ for $l>0$.

With this result the convolution (42) becomes

$$
I^{\prime}\left(Q_{m}\right)=\sum_{k=\max [-n,-m]}^{+n} I\left(Q_{m+k}\right) V\left(Q_{m}, Q_{m+k}\right)
$$

Figure 15 attempts to capture the essence of what is being performed here. Note that equations (A.2) and (A.3) rely on the fact that that the diameter of the top hat sphere is specified as a whole (odd) number of bin widths. Without this constraint the method would not work.

[1] L. Van Hove, Phys. Rev. 95, 249 (1954).

[2] G. Placzek, Phys. Rev. 86, 377 (1952).

[3] R. G. Sachs and E. Teller, Phys. Rev. 60, 18 (1941).

[4] T. J. Krieger and M. S. Nelkin, Phys. Rev. 106, 290 (1957).

[5] R. N. Sinclair and A. C. Wright, Nucl. Inst. Meth. 114, 451 (1974).

[6] G. Rickayzen and J. G. Powles, Mol. Phys. 32, 301 (1976).

[7] J. G. Powles and G. Rickayzen, Mol. Phys. 32, 323 (1976).

[8] J. G. Powles, Mol. Phys. 36, 1161 (1978).

[9] J. G. Powles, Mol. Phys. 36, 1181 (1978).

[10] T. Matsumoto, J. Nucl. Sci. Techn. 16, 401 (1979).

[11] J. G. Powles, Mol. Phys. 37, 623 (1979).

[12] P. A. Egelstaff and A. K. Soper, Mol. Phys. 40, 553 (1980).

[13] P. A. Egelstaff and A. K. Soper, Mol. Phys. 40, 569 (1980).

[14] J. L. Yarnell, M. J. Katz, R. G. Wenzel, and S. H. Koenig, Phys. Rev. A 7, 2130 (1973). 


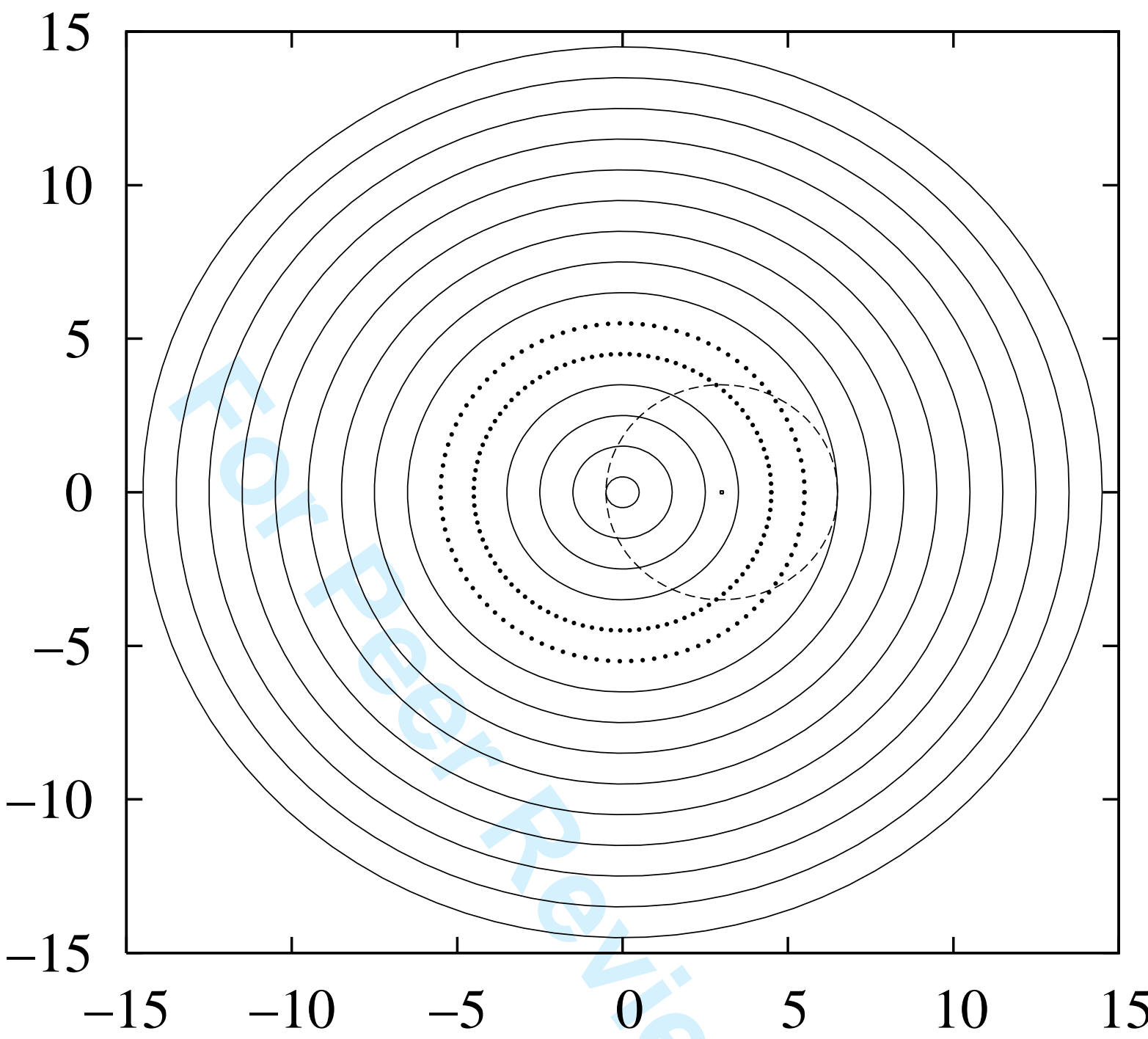

FIG. 15: 2-dimensional representation of the top hat convolution for discrete data. Equally spaced concentric rings of unit width are shown, corresponding to the 3-dimensional shells of reciprocal space. The rings represent the boundaries for each $Q$ bin. The top hat sphere $(n=3)$ is shown as a circle (dashed line) centred at $Q_{m}$ with $m=3$. The $m+k=5$ shell is highlighted (dotted boundaries), which means $k=+2$ in this case. After smearing, the intensity that occurs initially in the shell $m=3$ will be distributed to all the shells that occur inside the dashed line. The amount distributed into each neighbouring shell is in proportion to the volume of the top hat sphere which is enclosed by the shell boundaries. Hence for the case $k=+2$ this is the volume enclosed by the dotted lines that is also inside the dashed line sphere. Note that for $k=-3(m+k=0)$ the volume is simply that of a solid sphere of diameter 1 unit. 
[15] L. Blum and A. H. Narten, J. Chem. Phys. 64, 2804 (1976).

[16] L. Blum, M. Rovere, and A. H. Narten, J. Chem. Phys. 77, 2647 (1982).

[17] M. Rovere, L. Blum, and A. H. Narten, J. Chem. Phys. 73, 3729 (1980).

[18] J. R. Granada, Phys. Rev. B 31, 4167 (1985).

[19] J. R. Granada, V. H. Gillette, and R. E. Mayer, Physica B \& C 142, 223 (1986).

[20] P. A. Egelstaff, in Methods of Experimental Physics, Vol 23: Neutron scattering, Part B, edited by D. L. Price and K. Sköld (London and New York: Academic, 1987), chap. 14, pp. $405-470$.

[21] M. Misawa, D. L. Price, and K. Suzuki, J. Non-Cryst. Sol. 37, 85 (1980).

[22] J. R. Granada, V. H. Gillette, and R. E. Mayer, Physica B 156, 164 (1989).

[23] M. A. Howe, R. L. Mcgreevy, and W. S. Howells, J. Phys. Condens. Matter 1, 3433 (1989).

[24] A. K. Soper and A. Luzar, J. Chem. Phys. 97, 1320 (1992).

[25] J. Dawidowski, G. J. Cuello, and J. R. Granada, Nucl. Inst. Meth. Phys. Res. Sec. B - Beam Interactions with Materials and Atoms 82, 459 (1993).

[26] J. Dawidowski, J. R. Granada, R. E. Mayer, G. J. Cuello, V. H. Gillette, and M. C. Bellissentfunel, Physica B 203, 116 (1994).

[27] J. Dawidowski, J. R. Granada, G. J. Cuello, and V. H. Gillette, Physica B 213, 616 (1995).

[28] P. Postorino, M. Nardone, M. A. Ricci, and M. Rovere, J. Mol. Liq. 64, 221 (1995).

[29] Y. Kameda, M. Sasaki, T. Usuki, T. Otomo, K. Itoh, K. Suzuya, and T. Fukunaga, J. Neutron Res. 11, 153 (2003).

[30] H. E. Fischer, A. C. Barnes, and P. S. Salmon, Rep. Prog. Phys. 69, 233 (2006).

[31] A. C. Zemach and R. J. Glauber, Phys. Rev. 101, 118 (1956).

[32] C. Andreani, D. Colognesi, J. Mayers, G. F. Reiter, and R. Senesi, Adv. Phys. 54, 377 (2005).

[33] M. Abramovitz and I. A. Stegun, Handbook of Mathematical Functions, Applied Mathematics Series 55 (National Bureau of Standards, 1964), 1972nd ed.

[34] E. Bright Wilson Jr., D. J. C., and P. C. Cross, Molecular Vibrations - The Theory of Infrared and Raman Spectroscopy (Dover, New York, 1980).

[35] C. Windsor, Pulsed Neutron Scattering (Taylor and Francis, London, 1981).

[36] J. P. Hansen and I. R. McDonald, Theory of Simple Liquids (Academic Press, London, 1986), 2nd ed.

[37] E. Lorch, J. Phys. C (Solid St. Phys.) 2, 229 (1969). 
[38] A. K. Soper and C. Benmore, Phys. Rev. Lett. 101, 065502 (2008).

[39] P. Zetterstrom, A. K. Soper, and P. Schofield, Molec. Phys. 88, 1621 (1996).

[40] P. Zetterstrom, A. K. Soper, and P. Schofield, Molec. Phys. 90, 773 (1997). 


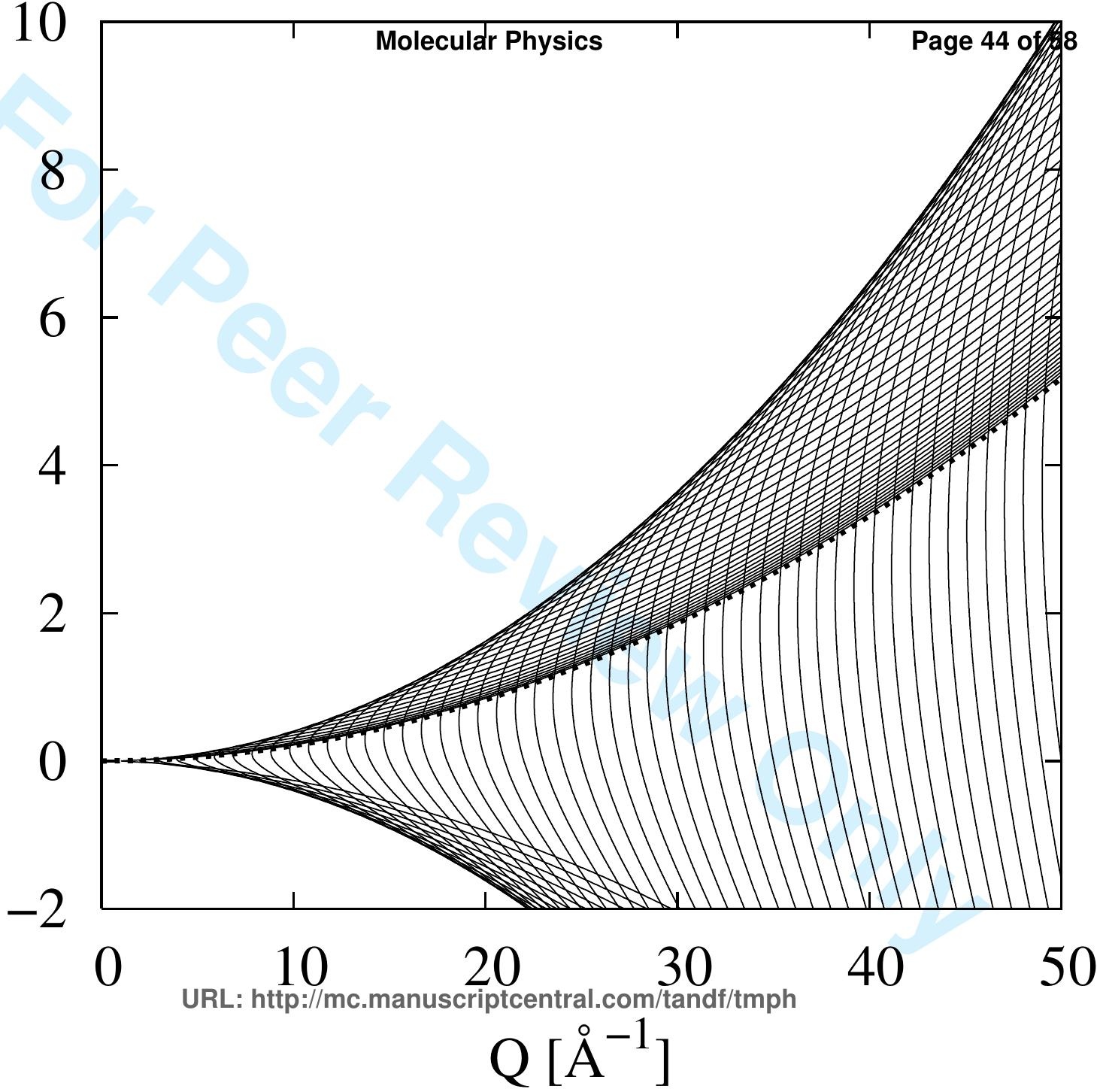




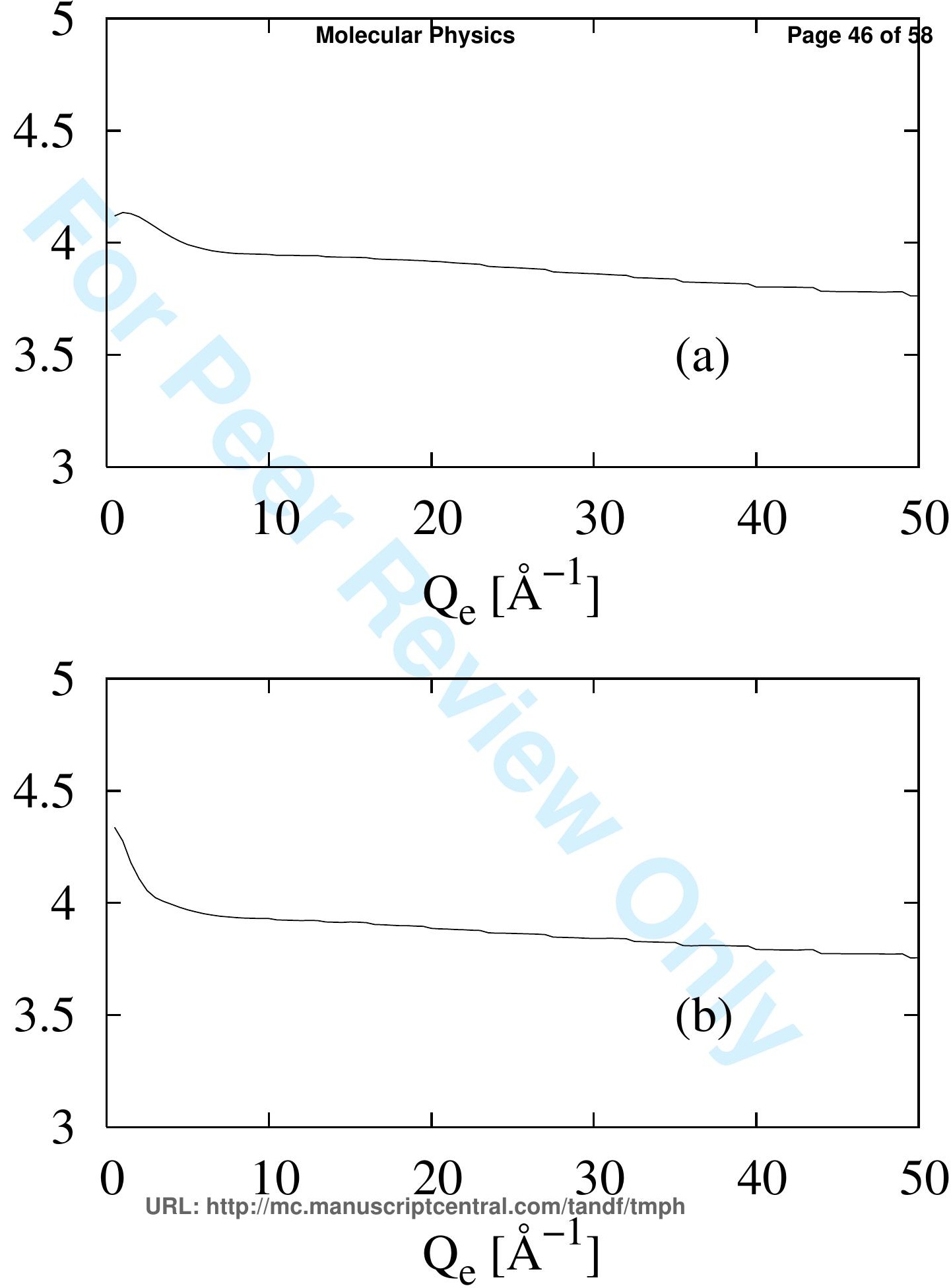




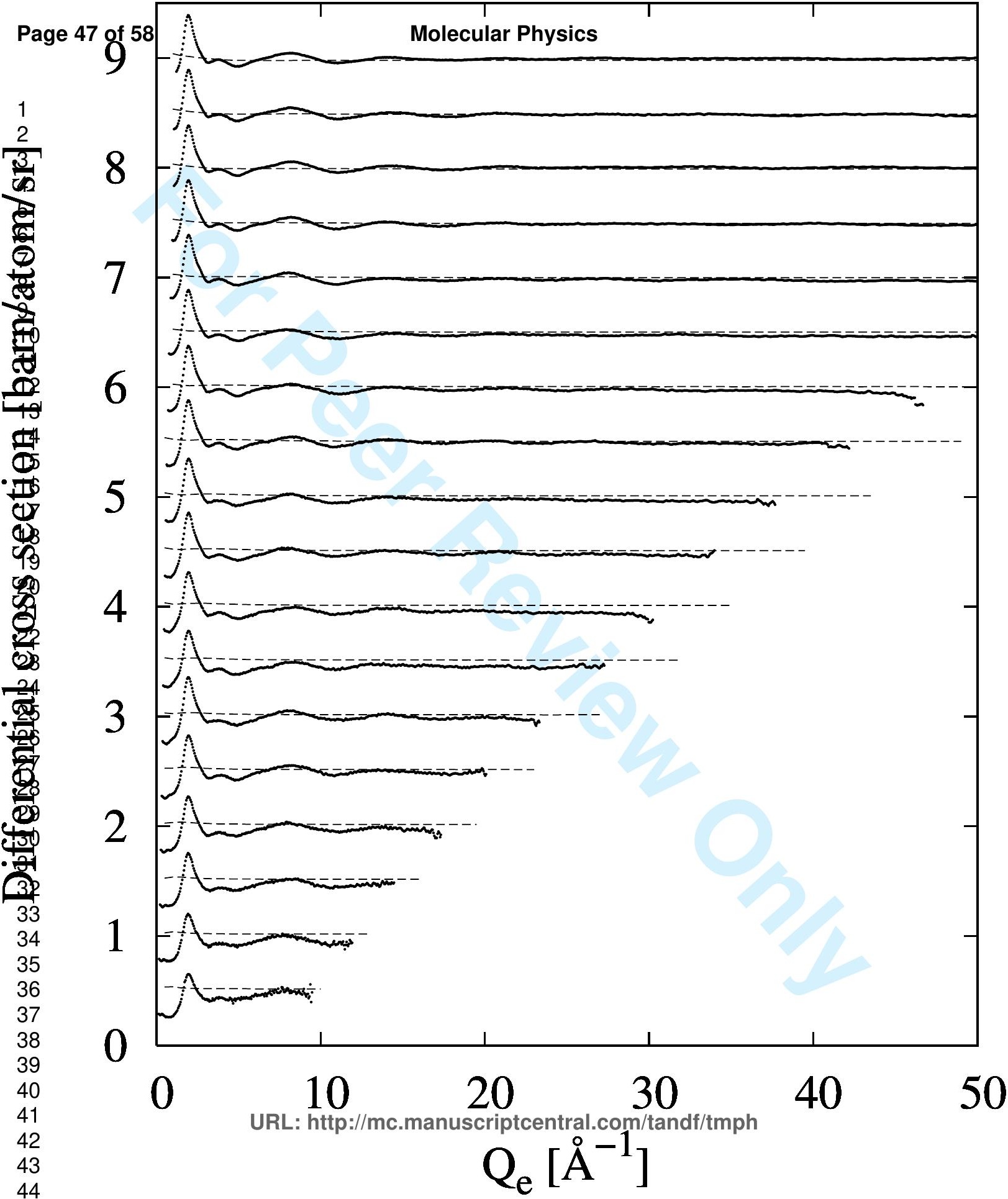




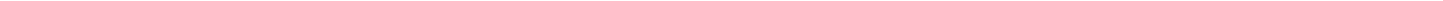




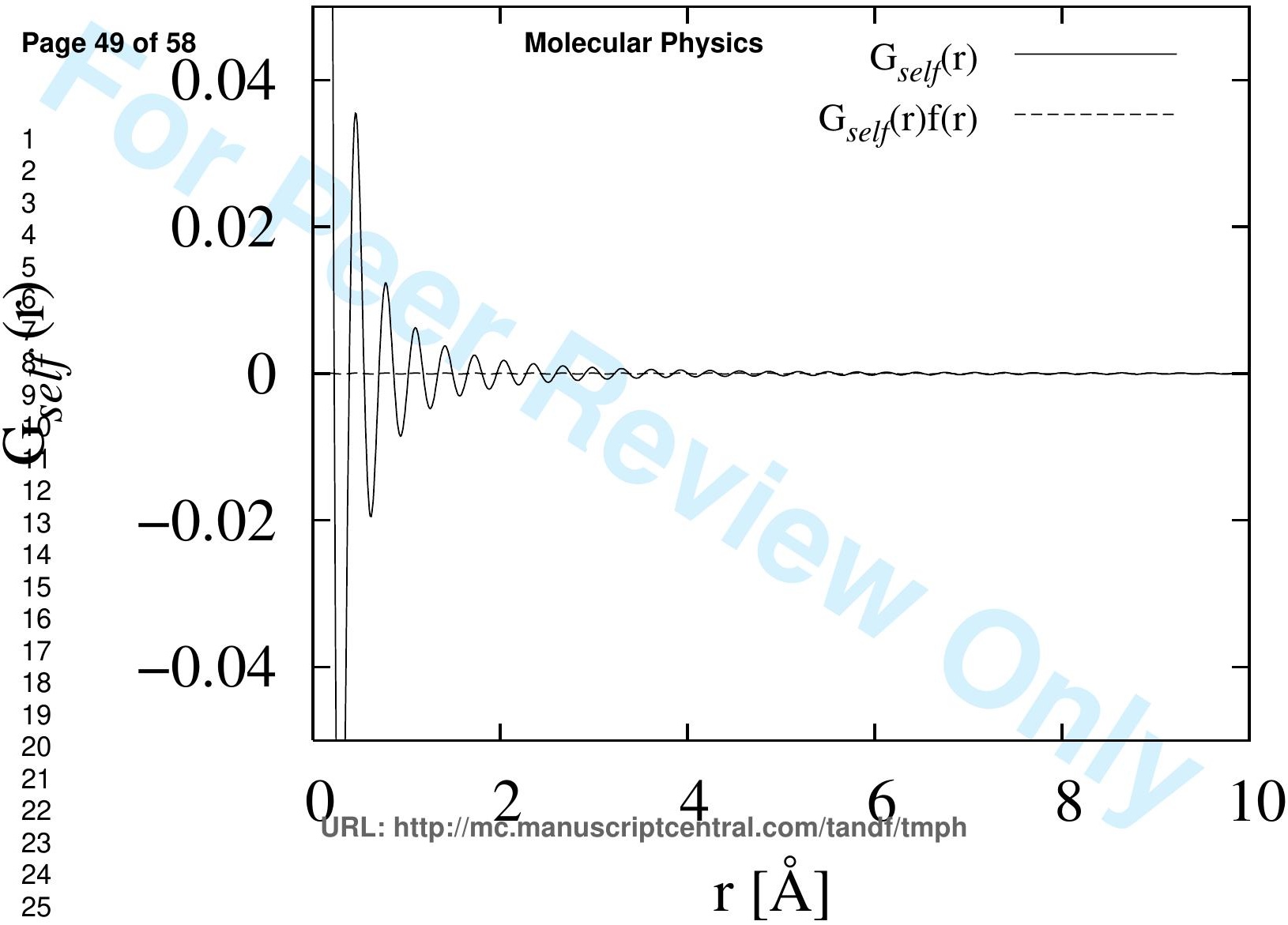




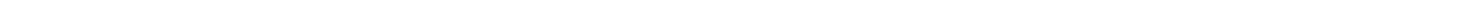




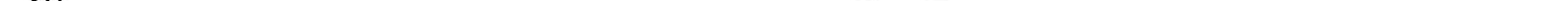




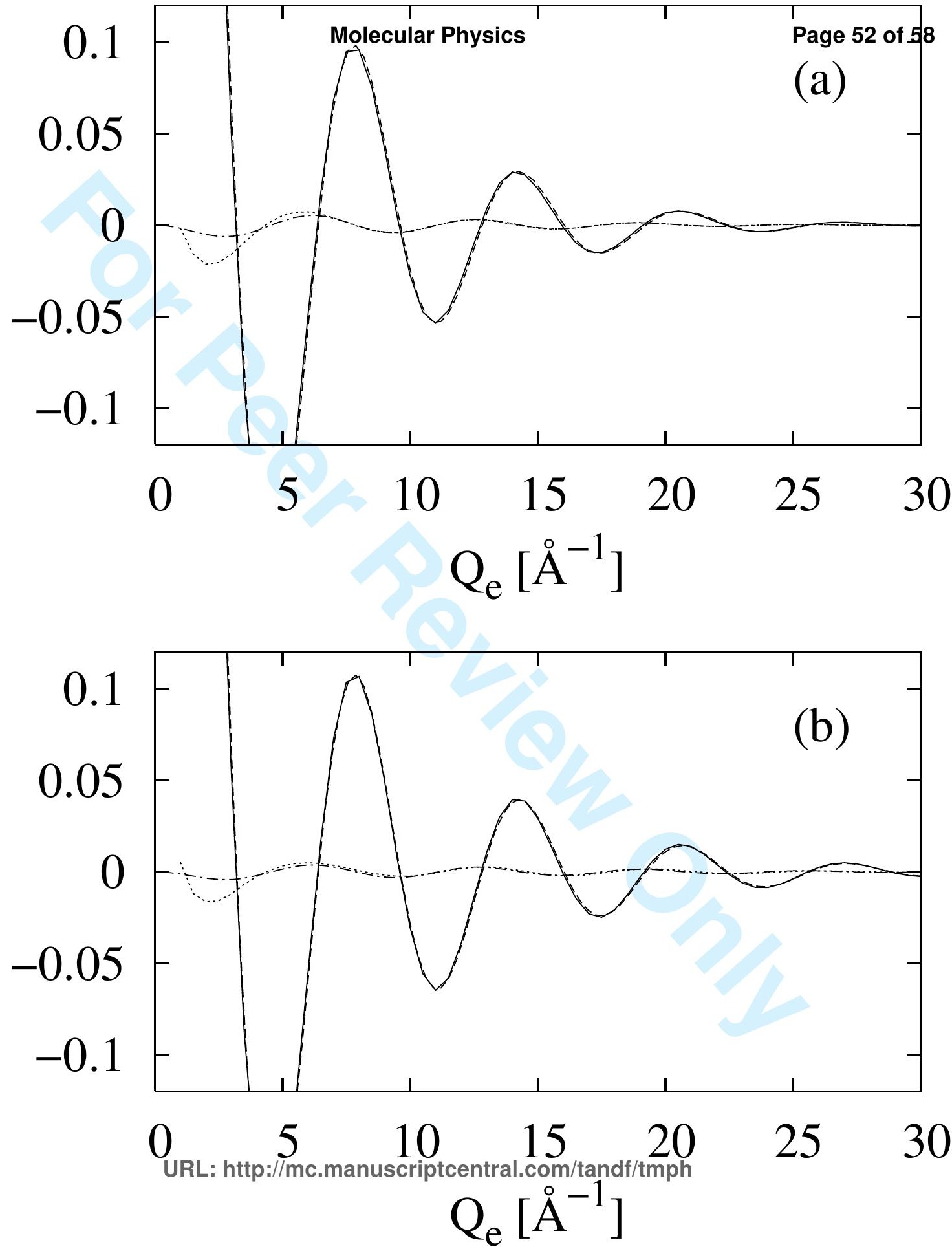




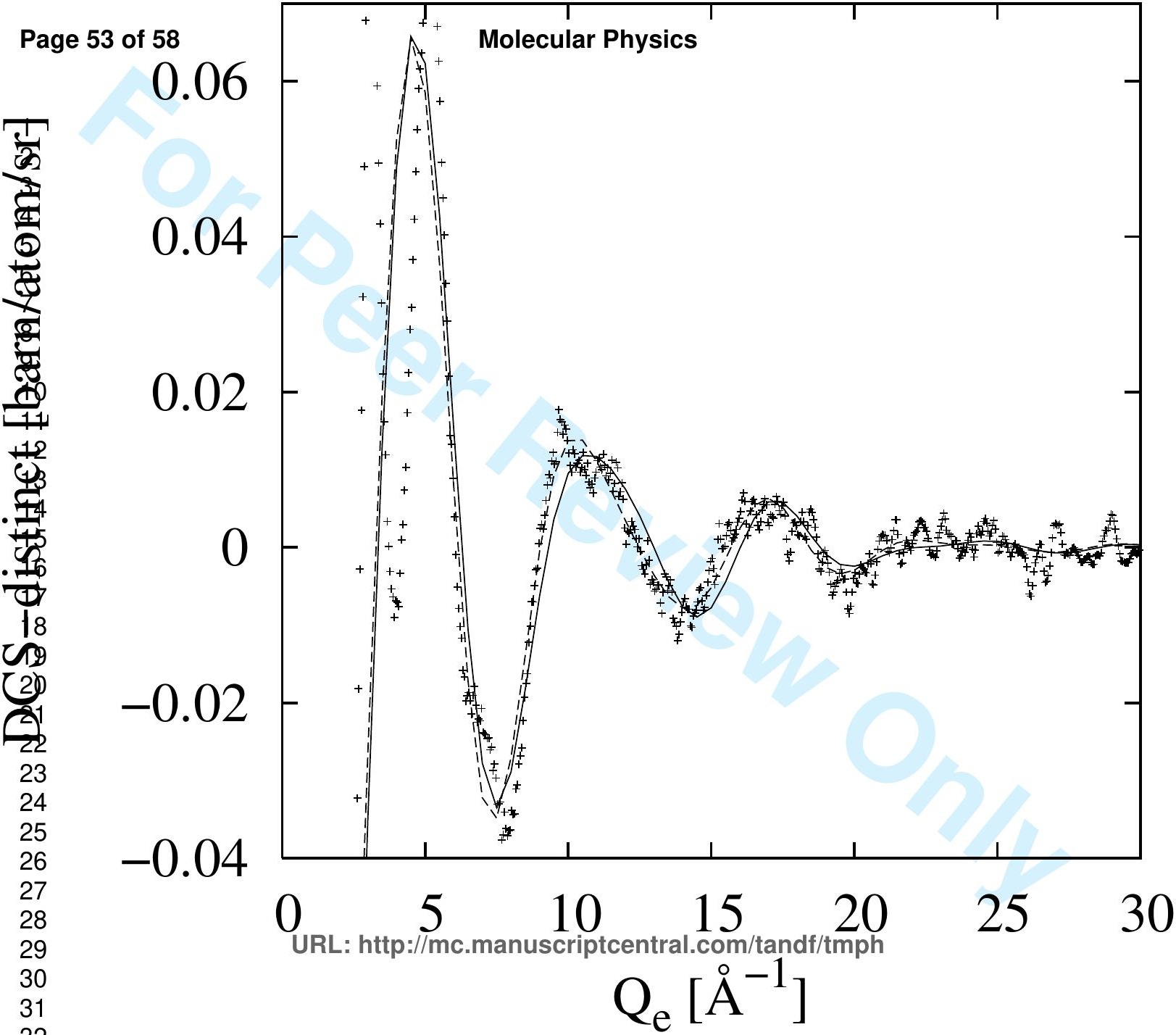




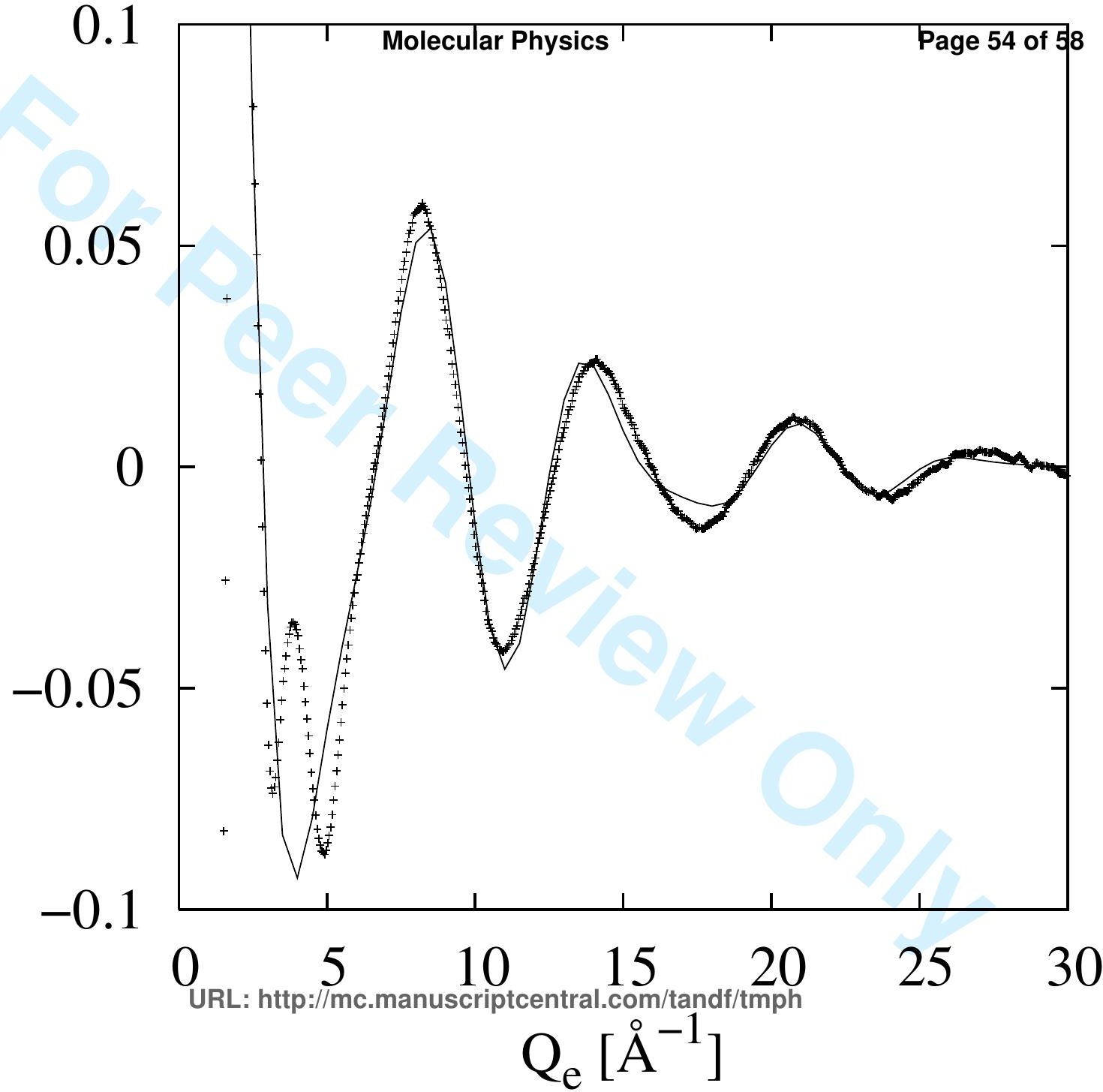




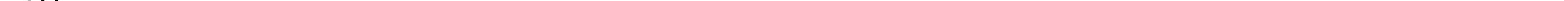




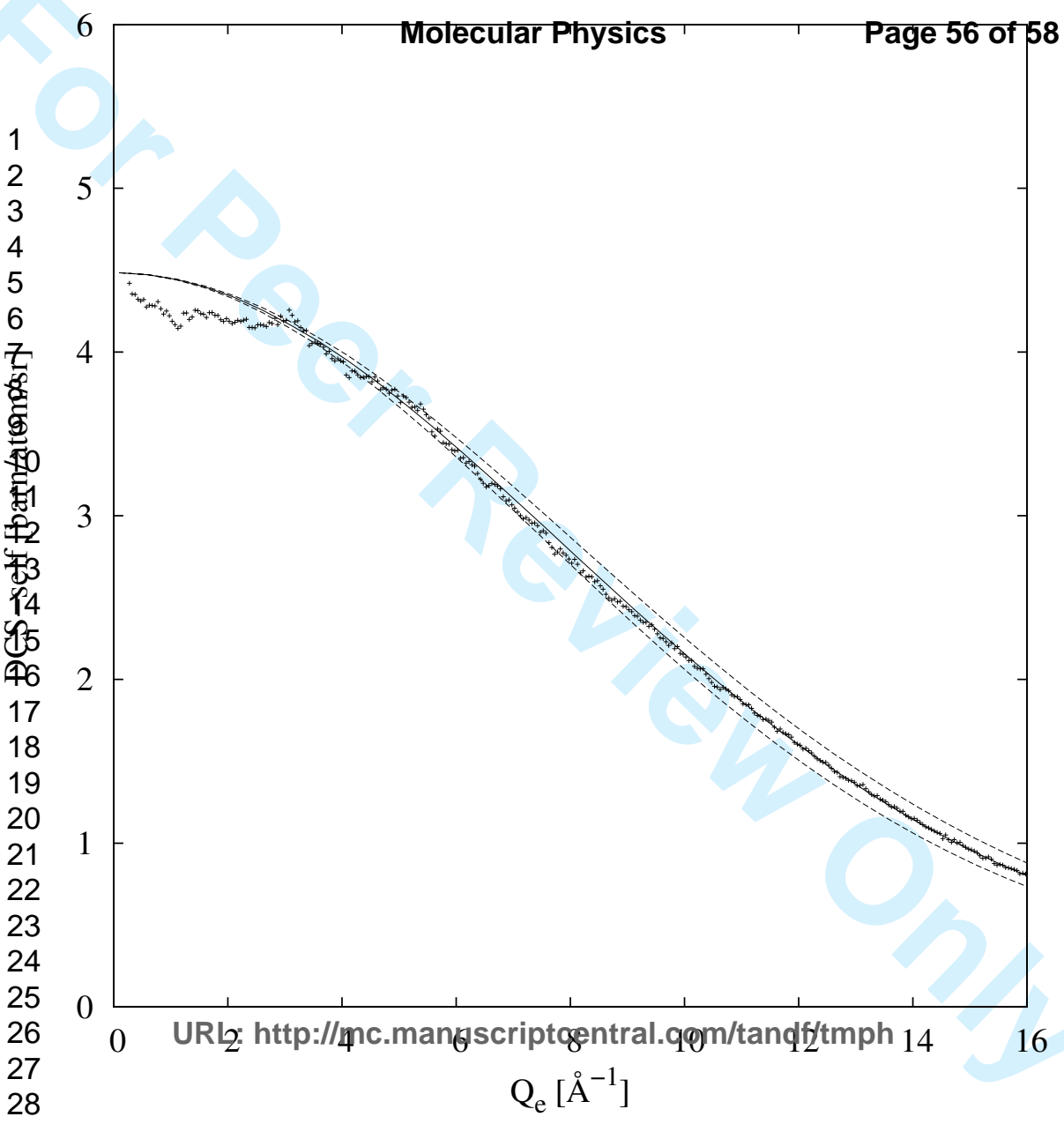




\section{Page 572 of 58}

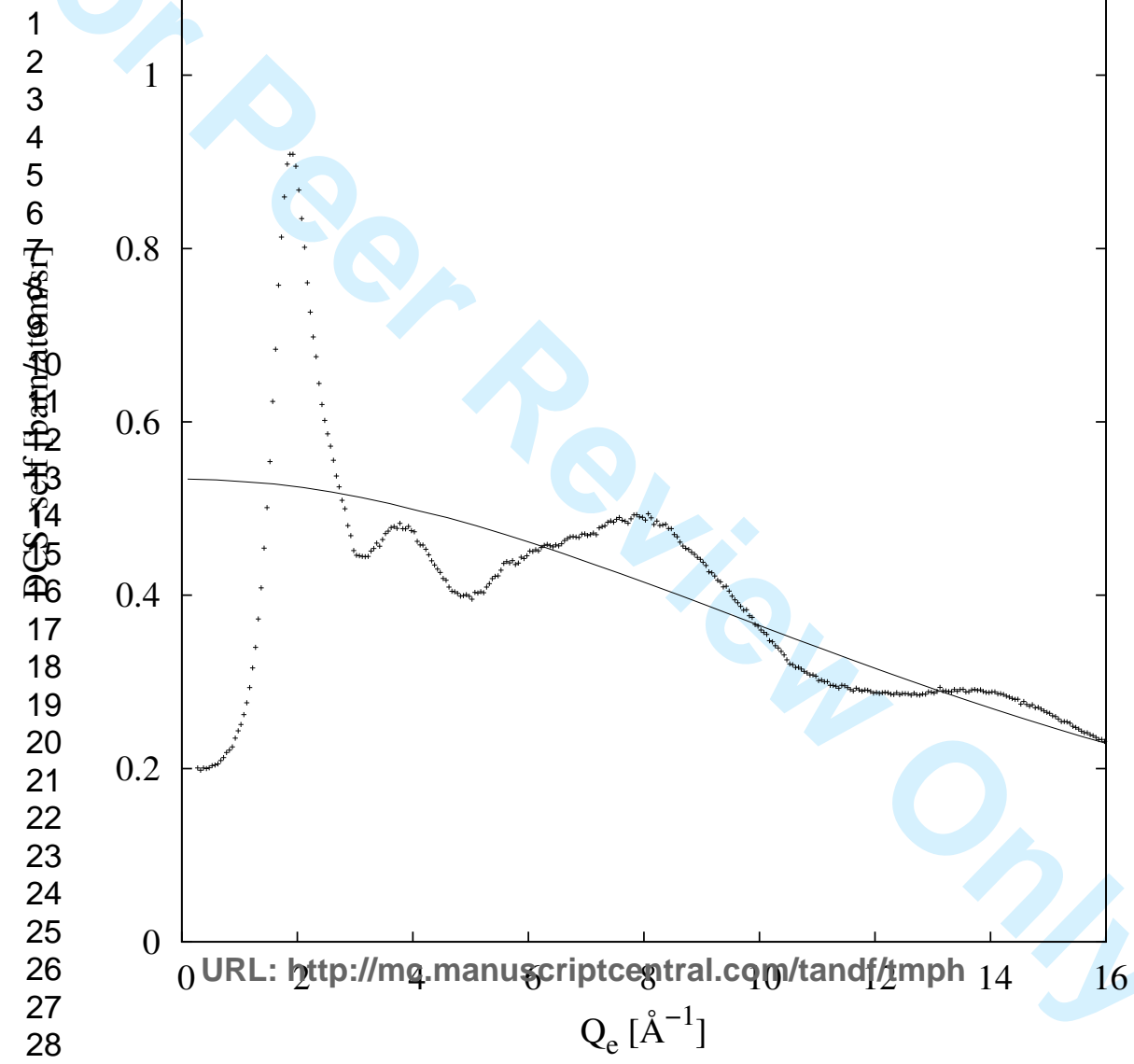




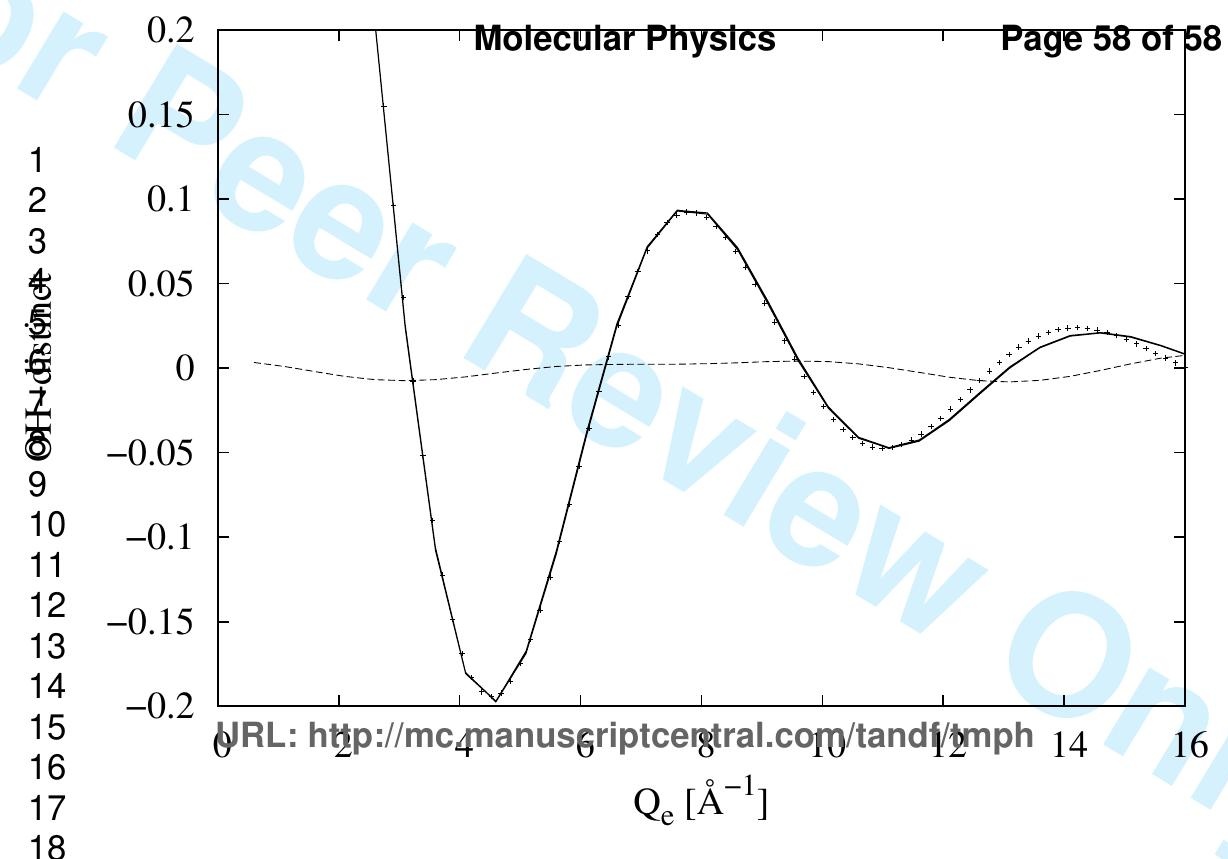

